Published by Cambridge University Press

This is an Open Access article, distributed under the terms of the Creative Commons Attribution

licence (http://creativecommons.org/licenses/by/4.0/), which permits unrestricted re-use, distribution,

and reproduction in any medium, provided the original work is properly cited.

doi:10.1017/jfm.2020.411

\title{
Bidispersive thermal convection with relatively large macropores
}

\author{
M. Gentile ${ }^{1, \dagger}$ and B. Straughan ${ }^{2}$ \\ ${ }^{1}$ Dipartimento di Matematica e Appl. 'R.Caccioppoli', Università degli Studi di Napoli Federico II, \\ Via Cintia, Monte S. Angelo, 80126 Napoli, Italy \\ ${ }^{2}$ Department of Mathematical Sciences, Durham University, DH1 3LE, UK
}

(Received 9 November 2019; revised 18 May 2020; accepted 20 May 2020)

We derive linear instability and nonlinear stability thresholds for a problem of thermal convection in a bidispersive porous medium with a single temperature when Darcy theory is employed in the micropores whereas Brinkman theory is utilized in the macropores. It is important to note that we show that the linear instability threshold is the same as the nonlinear stability one. This means that the linear theory is capturing completely the physics of the onset of thermal convection. The coincidence of the linear and nonlinear stability boundaries is established under general thermal boundary conditions.

Key words: convection in porous media, nonlinear instability, Bénard convection

\section{Introduction}

A bidispersive, or dual porosity, porous medium is one where the solid skeleton contains two types of pores. One type consists of the normal pores one recognizes, and these are the macropores. However, there are in addition micropores which may be cracks in the skeleton or may be deliberately created in a man made bidispersive material. For example, very small glass beads may be assembled to create an almost overall spherical 'raspberry like' shape, and these larger spheres then joined together form the bidispersive porous medium; see the picture given on page 3069 of Nield \& Kuznetsov (2006a). Hooman, Sauret \& Dahari (2015) describe a bidisperse porous medium consisting of thin plates and hot water pipes, while Imani \& Hooman (2017) deal with small blocks which are arranged into larger blocks, and other types of bidisperse porous media may be found in Straughan (2015, p. 7, p. 184) and Straughan (2017, pp. 2-8).

The porosity associated with the macropores is denoted by $\phi$, i.e. $\phi$ is the ratio of the volume of the macropores to the total volume of the saturated porous material. The micropores are responsible for a porosity $\varepsilon$ which is the ratio of the volume occupied by the micropores to the volume of the porous body which remains once the macropores are removed. This means the fraction of volume occupied by the

$\dagger$ Email address for correspondence: m.gentile@unina.it 
micropores is $\varepsilon(1-\phi)$ whereas the fraction of the volume occupied by the solid skeleton is $(1-\varepsilon)(1-\phi)$.

Dual-porosity porous media have been investigated in the chemical engineering and physics literature for some time. For example, Hashimoto \& Smith (1974), Kawazoe \& Takeuchi (1975) and Taqvi, Vishnoi \& Levan (1997) concentrate on the adsorption of fluid on surfaces and diffusion in a bidisperse porous medium. Guyon, Oger \& Plona (1994) present an experimental study of sintered bimodal distributions of spheres with size ratio $1: 4$, and determine empirical relations for the effective resistivity of the pore fluid, and for the permeability of the materials, as a function of the porosity. Gerke \& van Genuchten (1993a) present parabolic equations for the pressures of fluid in the fracture (micro) and in the matrix (macro) together with parabolic equations for solute transport in both regions. Simulations of these equations are performed using finite elements. Gerke \& van Genuchten (1993b) perform similar modelling and analysis for a dual-porosity medium which is not fully saturated. Gerke \& van Genuchten (1996) further the analysis of the above mentioned studies and use various geometrical shapes for the matrix geometries. Other references to the chemical engineering literature are provided in Chabanon, David \& Goyeau (2015).

It would appear that temperature effects in bidisperse porous media were first investigated by Chen, Cheng \& Zhao (2000b) and Chen, Cheng \& Hsu (2000a). Chen et al. (2000b) present results of an experimental study of channels packed with sintered copper bidispersed porous media. They showed that the bidisperse porous medium is a highly effective two-phase heat sink. Chen et al. (2000a) present measurements of two samples of bidisperse porous media saturated with three different fluids. They find that the effective thermal conductivity of a bidisperse porous medium is smaller than that of the equivalent monodispersed porous medium.

Specific theoretical approaches of fluid flow in isothermal dual-porosity media appear to have begun with Vernescu (1990) who employed Stokes flow in the fluid and Darcy theory for flow in porous blocks immersed (and held fixed) in the fluid. He identified three regimes where the overall flow could be described by Darcy theory, Brinkman theory or by Stokes flow, depending on the characteristic length of the porous obstacles and the distance between these. Moutsopoulos \& Koch (1999) developed a model for flow in a bidisperse porous medium by assuming the smaller grains only affect the flow through their permeability $K_{2}$. They use a Brinkman theory to model flow in a bidisperse porous medium with one velocity field. Their equations governing incompressible flow in a bidisperse porous medium have the form

$$
-\mu \Delta u_{i}+p_{, i}+\frac{\mu}{K_{2}} u_{i}=0, \quad u_{i, i}=0,
$$

where $\mu$ is the fluid viscosity, $p$ pressure and $u_{i}$ the velocity in the porous medium. The study of Moutsopoulos \& Koch (1999) concentrates on being able to follow diffused material in the porous medium and to this end they couple equations (1.1) with an equation for the tracer concentration, $c$,

$$
\frac{\partial c}{\partial t}+\left(v_{i} c\right)_{, i}=D \Delta c
$$

where $v_{i}=u_{i} /\left(1-\phi_{2}\right), \phi_{2}$ being the volume fraction of the smaller particles, and $D$ is the diffusion coefficient. Throughout we employ standard indicial notation in 
conjunction with the Einstein summation convention. Thus, a repeated index sums from 1 to 3 whereas a free index may take the values 1,2 or 3 . For example,

$$
u_{i, i} \equiv \sum_{i=1}^{3} \frac{\partial u_{i}}{\partial x_{i}}=\frac{\partial u_{1}}{\partial x}+\frac{\partial u_{2}}{\partial y}+\frac{\partial u_{3}}{\partial z}
$$

or

$$
\lambda_{i j} u_{j} \equiv \sum_{j=1}^{3} \lambda_{i j} u_{j}=\lambda_{i 1} u_{1}+\lambda_{i 2} u_{2}+\lambda_{i 3} u_{3}, \quad i=1,2 \text { or } 3
$$

for some tensor $\lambda_{i j}$, or

$$
\left(v_{i} c\right)_{, i} \equiv \sum_{i=1}^{3} \frac{\partial}{\partial x_{i}}\left(v_{i} c\right)=\frac{\partial}{\partial x}\left(c v_{1}\right)+\frac{\partial}{\partial y}\left(c v_{2}\right)+\frac{\partial}{\partial z}\left(c v_{3}\right) .
$$

A key development in the theory of bidisperse porous media is due to Nield \& Kuznetsov (2005) who develop and utilize a novel model which employs different velocities $U_{i}^{f}$ and $U_{i}^{p}$ in the macro and micropores, different pressures $p^{f}$ and $p^{p}$ and additionally different temperatures $T^{f}$ and $T^{p}$. Throughout we use $f$ to denote macro and $p$ to denote microquantities. This model was explicitly adapted to thermal convection in Nield \& Kuznetsov (2006a), where they used Brinkman theory for both the macro and microphases and presented a linear instability analysis. A global nonlinear energy stability analysis for the same problem was given by Straughan (2009), who derived nonlinear stability Rayleigh numbers which were lower than the linear instability ones of Nield \& Kuznetsov (2006a). Straughan (2015, pp. 183-202) developed both linear instability and global nonlinear stability analyses for thermal convection in a Nield-Kuznetsov bidisperse porous medium when Darcy theory was employed in both the macro and microphases. In that work there is a large gap between the critical Rayleigh numbers of linear theory and those for the nonlinear threshold. While this does not imply the existence of sub-critical instabilities, it does leave open the possibility. Also, exchange of stabilities has not been proved in either the Brinkman-Brinkman or Darcy-Darcy case when there are two temperatures and so there is a possibility of oscillatory convection in addition to the already observed stationary convection.

The two velocity theory of Nield \& Kuznetsov $(2005,2006 a)$ has been successfully applied to many problems of forced convection, numerical simulation and other flows by Nield \& Kuznetsov (2004, 2006b, 2007, 2008, 2011, 2013), Rees, Nield \& Kuznetsov (2008b), Kumari \& Pop (2009), Revnic et al. (2009), Narasimhan \& Reddy (2011a,b), Narasimhan, Reddy \& Dutta (2012), Magyari (2013), Nield \& Bejan (2013), Nield (2016), Wang et al. (2017), Wang \& Li (2018) and Patrulescu, Grosan \& Pop (2020).

Two very important articles pertaining to the theory of Nield \& Kuznetsov (2006a) are those of Hooman et al. (2015), and Imani \& Hooman (2017). The work of Hooman et al. (2015) analyses heat transfer in a plate-fin heat exchanger and they perform the first calculation of values for the momentum transfer coefficient for flow between the macro and microphases. This is a key parameter in the Nield \& Kuznetsov (2006a) theory and previously no values were known. The work of Imani \& Hooman (2017) uses a bidisperse porous medium consisting of blocks of porous material which are themselves composed of smaller microblocks. They note that the 
theory of Nield \& Kuznetsov (2006a) involves two unknown parameters, namely the heat transfer coefficient and the interfacial momentum transfer coefficient, and they also point out that the theory relies on the assumption of local thermal equilibrium within the microporous medium. The heat transfer coefficient issue is addressed by Rees $(2009,2010)$, and the interfacial momentum concern was addressed by Hooman et al. (2015), as stated above. However, the local thermal issue is resolved in Imani \& Hooman (2017). They show that when the macropores are relatively large compared to the micropores then one may assume the temperatures of the solid skeleton match those of the fluid in the macro and microphases, i.e. there is local thermal equilibrium. Precisely, they write ... 'it is shown that departure from the local thermal equilibrium condition is observed for the higher values of the Rayleigh number, microporous porosity, solid-fluid thermal conductivity ratio, and the smaller values of the macropores volume fraction'.

When large temperature differences are expected in the macro and micropores the two temperature theory should be used, for example when hot fluid is injected into a cold skeleton, see Rees, Bassom \& Siddheshwar (2008a), Rees \& Bassom (2010). However, for many applications a single temperature, $T$, may suffice. With this in mind Falsaperla, Mulone \& Straughan (2016) and Gentile \& Straughan (2017a) adapted the Nield \& Kuznetsov (2006a) theory to a single temperature. This reduced theory has subsequently been successfully employed in a variety of contexts by Capone, De Luca \& Gentile (2020), Gentile \& Straughan (2017b) and Straughan $(2018,2019 a, b)$. All of the just cited articles employing a single temperature assume the flow in both the macro and microphases is described by Darcy theory.

The configuration of blocks which are themselves smaller blocks of solid allow Imani \& Hooman (2017) to employ Navier-Stokes theory in the fluid with a regular heat equation in the solid skeleton. They use lattice-Boltzmann theory to justify the local thermal equilibrium (LTE) theory of Nield \& Kuznetsov (2006a). This gives reason for us to employ LTE theory throughout the bidisperse porous medium. From the mathematical point of view, using LTE theory, i.e. a single temperature, modifies the problem so we are able to show that exchange of stabilities holds, and we also show that the linear instability threshold is the same as the global nonlinear one. Thus, linear instability theory correctly captures the onset of thermal convection. We have not been able to prove such a result when two temperatures are present.

In this paper we allow for the possibility of larger pores where we employ a Brinkman theory, while still retaining Darcy theory in the micropores. We believe this is the first time a mixed Darcy-Brinkman model has been employed in bidispersive flow. It is worth noting that the original model of Nield \& Kuznetsov (2006a) employed a Brinkman theory in both the macro and micropores. Thermal convection in the two temperature bidispersive theory with Brinkman effects in both the macro and microphases, or with Darcy effects in both phases, is discussed at length in chapter 13 of Straughan (2015).

It is very important to realize that the Brinkman theory, even in a single-porosity medium, will yield very different stability bounds to those found with Darcy theory, see the detailed analysis of Rees (2002). Furthermore, Straughan (2016) has shown that Darcy theory may lead to oscillatory convection in a resonance problem whereas the equivalent Brinkman problem yields stationary convection. Hence, Darcy and Brinkman theory can lead to different physical effects.

We believe the use of Brinkman theory for the macrophase is justified since we are envisaging a material with relatively large macropores, i.e. a relatively large macroporosity. Givler \& Altobelli (1994) justify use of Brinkman theory when the 
porous medium is an open cell rigid foam, porosity $\phi=0.972$ and Durlofsky \& Brady (1987) achieve a similar justification for $\phi \geqslant 0.95$. Rubinstein (1986a) establishes a similar justification when $\phi \geqslant 0.8$; Rubinstein (1986b) proves a beautiful result whereby he gives a rigorous proof of the convergence of Stokes' equation in domains containing a random distribution of identical spheres to Brinkman's equation. The Brinkman equation in bidisperse porous media is employed by Moutsopoulos \& Koch (1999). Chabanon et al. (2015) to define three scales for a bidisperse porous material, a macroscopic scale, a microscopic scale and also a mesoscopic scale. They use averaging techniques in the mesoscopic scale and show the relevant equation is one of Brinkman form where the Navier-Stokes Laplacian term and the Darcy linear term are simultaneously present. They present numerical results for a bidisperse material which is composed of staggered cylinders at both macroscopic and microscopic scales with a porosity of 0.6 , which is in the same range as that noted by Nield (2000, p. 168).

Bidisperse porous media are being increasingly employed in industry or are being recognized as being key to many real life processes and so we believe understanding a macro-Brinkman-micro-Darcy model is essential. The application areas for double-porosity media (bidispersive) are very varied and include for example, chemical engineering technology, see e.g. Enterria et al. (2014); coal stockpiling, see Hooman \& Maas (2014); gas shale storage, see Alnoaimi \& Kovscek (2019); heat pipe technology, see Lin et al. (2011) and Mottet \& Prat (2016); hydraulic fracturing of subterranean rocks for natural gas, see e.g. Jiang et al. (2018), Kim \& Moridis (2015) and Zhang et al. (2019); understanding landslides, see e.g. Borja, Liu \& White (2012) and Montrasio, Valentino \& Losi (2011); and in particular with reference to the caldera in the Campi Flegrei in the area to the west of Naples, see Scotto di Santolo \& Evangelista (2008).

It is important to include temperature effects in bidispersive flow because thermal stresses are likely to induce cracking in the solid skeleton and this produces micropores, cf. Homand-Etienne \& Houpert (1989), Gelet, Loret \& Khalili (2012) and Kim \& Hosseini (2015).

In this work we derive a model for thermal convection in a bidisperse porous medium with one temperature field allowing for Brinkman effects in the macropores whilst retaining only Darcy effects in the micropores. Thermal convection is analysed in a horizontal porous layer and we prove the strong result that the linear instability threshold is exactly the same as the global nonlinear stability boundary obtained from energy stability theory. This result is proved for anisotropic macro and micropermeabilities although we restrict our attention to the physically important case of symmetric permeability tensors. In addition, we allow for general thermal boundary conditions encompassing the case of radiation and a prescribed temperature. We also include a detailed account of numerical results in the prescribed temperature case when the permeabilities are isotropic.

\section{Governing equations in the isotropic case}

A general theory for bidisperse porous media is presented in Franchi, Nibbi \& Straughan (2017). These writers combine the model of Nield \& Kuznetsov (2006a) for a bidisperse porous medium together with the local thermal non-equilibrium equations for a monodisperse porous material of Nield (1998) and Banu \& Rees (2002), to derive a theory which involves macro and micropore averaged velocities, an individual solid skeleton, macro and micro temperature fields. This may be seen as follows. 


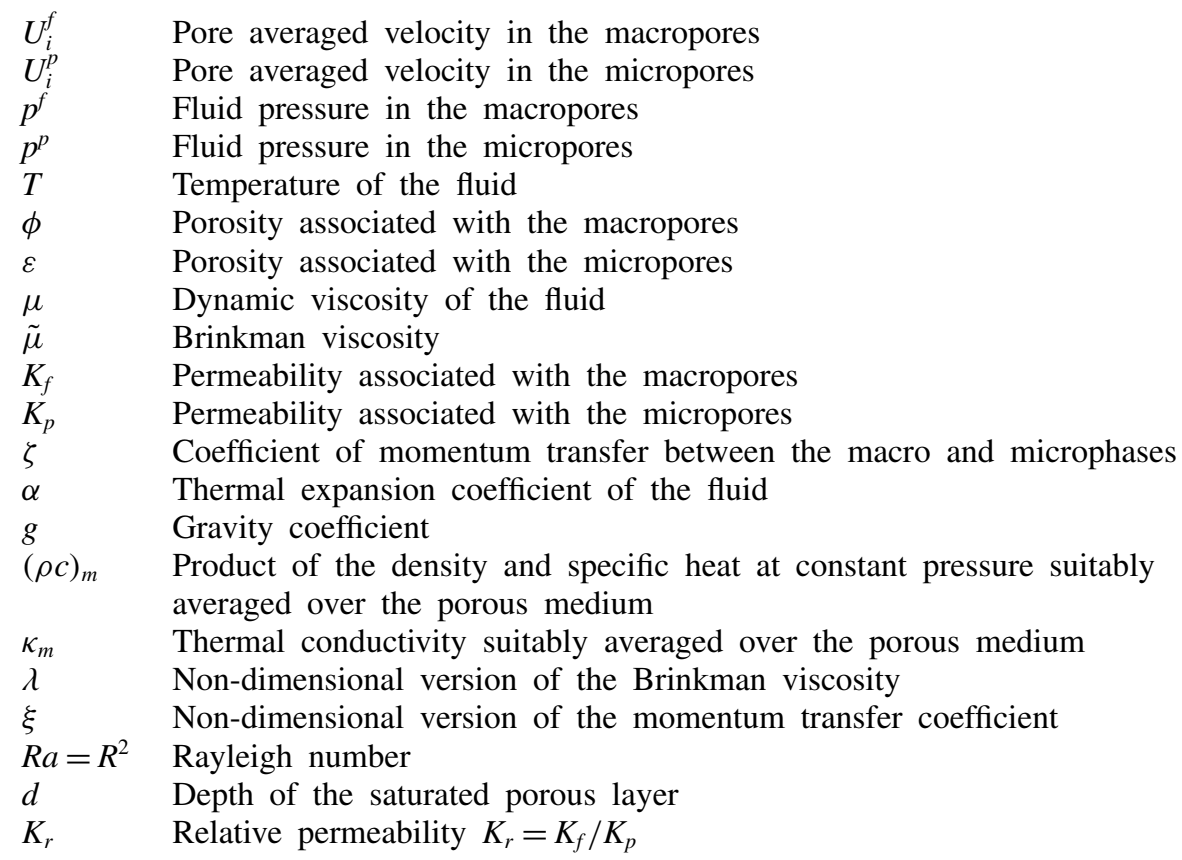

TABLE 1. List of nomenclature.

For an isotropic bidisperse porous medium the momentum and continuity equations of Nield \& Kuznetsov (2006a) may be written

$$
\left.\begin{array}{l}
-\frac{\mu}{K_{f}} U_{i}^{f}+\tilde{\mu} \Delta U_{i}^{f}-\zeta\left(U_{i}^{f}-U_{i}^{p}\right)-p_{, i}^{f} \\
\quad+\rho_{F} g \alpha k_{i}\left[\frac{\phi}{\phi+\varepsilon(1-\phi)} T^{f}+\frac{\varepsilon(1-\phi)}{\phi+\varepsilon(1-\phi)} T^{p}\right]=0, \\
U_{i, i}^{f}=0, \\
-\frac{\mu}{K_{p}} U_{i}^{p}-\zeta\left(U_{i}^{p}-U_{i}^{f}\right)-p_{, i}^{p} \\
\quad+\rho_{F} g \alpha k_{i}\left[\frac{\phi}{\phi+\varepsilon(1-\phi)} T^{f}+\frac{\varepsilon(1-\phi)}{\phi+\varepsilon(1-\phi)} T^{p}\right]=0, \\
U_{i, i}^{p}=0,
\end{array}\right\}
$$

where $k_{i}=\delta_{i 3}$ and where the divergence free conditions (continuity equations) express the fact that the fluid is incompressible. Nield \& Kuznetsov (2006a) additionally include a Laplacian (Brinkman) term in equation $(2.1)_{3}$ but we only use Darcy theory in the microphase. In these equations $U_{i}^{f}, U_{i}^{p}, p^{f}, p^{p}, T^{f}$ and $T^{p}$ are the pore averaged velocities, pressure and temperature fields in the macro (f) phase and micro (p) phase, respectively. The quantities $\mu, \tilde{\mu}, \rho, K_{f}$ and $K_{p}$ denote the dynamic viscosity of the fluid, the Brinkman viscosity, the fluid density, the macropermeability and the micropermeability. In addition, $g, \alpha$ and $\rho_{F}$ denote gravity, thermal expansion coefficient of the fluid and a constant reference density, since in the buoyancy terms a Boussinesq approximation is employed to write the fluid density as a linear function of $T^{f}$ and $T^{p}$. Finally, $\zeta$ is a coefficient which represents the momentum transfer 
between the macro and microphases. All the variables and parameters defined above are listed in table 1.

In the solid skeleton, the macrophase and in the microphase the energy balances may be separately written to account for interactions between the temperatures of each phase. Taking into account the volume fraction occupied by each phase the energy balances are written as

$$
\left.\begin{array}{c}
\epsilon_{1}(\rho c)_{s} T_{, t}^{s}=\epsilon_{1} \kappa_{s} \Delta T^{s}+s_{1}\left(T^{f}-T^{s}\right)+s_{2}\left(T^{p}-T^{s}\right), \\
\phi(\rho c)_{f} T_{, t}^{f}+\phi(\rho c)_{f} V_{i}^{f} T_{, i}^{f}=\phi \kappa_{f} \Delta T^{f}+h\left(T^{p}-T^{f}\right)+s_{1}\left(T^{s}-T^{f}\right), \\
\epsilon_{2}(\rho c)_{p} T_{, t}^{p}+\epsilon_{2}(\rho c)_{p} V_{i}^{p} T_{, i}^{p}=\epsilon_{2} \kappa_{p} \Delta T^{p}+h\left(T^{f}-T^{p}\right)+s_{2}\left(T^{s}-T^{p}\right),
\end{array}\right\}
$$

where

$$
\epsilon_{1}=(1-\varepsilon)(1-\phi), \quad \epsilon_{2}=(1-\phi) \varepsilon,
$$

and where $s, f$ and $p$ denote solid skeleton, macrophase, microphase, $\rho c$ denotes the product of the respective density and specific heat at constant pressure and $\kappa$ denotes the thermal diffusivity. In (2.2) the velocities $V_{i}^{f}$ and $V_{i}^{p}$ are the actual velocities of the fluid which would be witnessed in an individual element in each phase. The coefficient $h$ is analogous to the $h$ of local non-thermal equilibrium theory as in Banu $\&$ Rees (2002), or in Rees $(2009,2010)$. The coefficients $s_{1}$ and $s_{2}$ represent thermal interactions between the solid skeleton and the macro and microphases.

In the present work we deal with a local thermal equilibrium theory and take the temperatures $T^{s}, T^{f}$ and $T^{p}$ to be the same, namely $T=T^{s}=T^{f}=T^{p}$. In this way we may follow the procedure advocated by Joseph $(1976$, p. 55) for a monodisperse porous material and we derive the governing system of equations from (2.1) and (2.2) as

$$
\left.\begin{array}{c}
\tilde{\mu} \Delta U_{i}^{f}-\frac{\mu}{K_{f}} U_{i}^{f}-\zeta\left(U_{i}^{f}-U_{i}^{p}\right)-p_{, i}^{f}+\rho_{F} \alpha g T k_{i}=0, \\
U_{i, i}^{f}=0, \\
-\frac{\mu}{K_{p}} U_{i}^{p}-\zeta\left(U_{i}^{p}-U_{i}^{f}\right)-p_{, i}^{p}+\rho_{F} \alpha g T k_{i}=0, \\
U_{i, i}^{p}=0, \\
(\rho c)_{m} T_{, t}+(\rho c)_{f}\left(U_{i}^{f}+U_{i}^{p}\right) T_{, i}=\kappa_{m} \Delta T .
\end{array}\right\}
$$

Here, $U_{i}^{f}$ and $U_{i}^{p}$ are the pore averaged velocities, namely $U_{i}^{f}=\phi V_{i}^{f}, U_{i}^{p}=\varepsilon(1-\phi) V_{i}^{p}$ and $(\rho c)_{m}$ and $\kappa_{m}$ are given by

$$
\begin{gathered}
(\rho c)_{m}=(1-\phi)(1-\epsilon)(\rho c)_{s}+\phi(\rho c)_{f}+\epsilon(1-\phi)(\rho c)_{p}, \\
\kappa_{m}=(1-\phi)(1-\epsilon) \kappa_{s}+\phi \kappa_{f}+\epsilon(1-\phi) \kappa_{p} .
\end{gathered}
$$

As we remarked in the introduction, Imani \& Hooman (2017) have provided a justification for considering the temperatures to have the same value $T$. The theory of Nield \& Kuznetsov (2006a) allows for different temperatures in the macro and micropores, but for problems involving the onset of thermal convection, since it is the same fluid in each type of pore, the thermal properties are the same and we believe this justifies the use of a single temperature. Note that we are assuming a relatively large porosity in the macrostructure and hence equation (2.3) $)_{1}$ includes a Brinkman term, but we only require Darcy theory at the microlevel since the porosity there may be smaller. We believe that this is the first study of flow in bidisperse porous media with (2.3). 


\section{General theory for thermal convection}

We suppose now the bidisperse porous medium is contained in the horizontal layer $0<z<d$. In order to describe thermal convection we allow a generalization of (2.3) to the situation where the bidisperse porous medium may be anisotropic, which results in the permeabilities and mass transfer coefficients being second-order tensors. The problem of convection in an anisotropic bidisperse porous medium which employs Darcy theory in both the macro and microphases has led to some novel convection cell behaviour, see Straughan $(2018,2019 a, b)$. Our first goal is to prove a general result showing coincidence of the linear instability and nonlinear stability boundaries and this we can do in a general anisotropic setting.

The relevant equations from (2.3) allowing for the solid skeleton to have an anisotropic structure have the form

$$
\left.\begin{array}{c}
\tilde{\mu} \Delta U_{i}^{f}-M_{i j}^{f} U_{j}^{f}-\zeta_{i j}\left(U_{j}^{f}-U_{j}^{p}\right)-p_{, i}^{f}+\rho_{F} \alpha g T k_{i}=0, \\
U_{i, i}^{f}=0, \\
-M_{i j}^{p} U_{j}^{p}-\zeta_{i j}\left(U_{j}^{p}-U_{j}^{f}\right)-p_{, i}^{p}+\rho_{F} \alpha g T k_{i}=0, \\
U_{i, i}^{p}=0, \\
(\rho c)_{m} T_{, t}+(\rho c)_{f}\left(U_{i}^{f}+U_{i}^{p}\right) T_{, i}=\kappa_{m} \Delta T .
\end{array}\right\}
$$

The term $\tilde{\mu}$ is the Brinkman viscosity, $\Delta$ is the Laplace operator and $M_{i j}^{f}$, $M_{i j}^{p}$ are symmetric tensors given by

$$
M_{i j}^{f}=\mu\left(K_{i j}^{f}\right)^{-1}, \quad M_{i j}^{p}=\mu\left(K_{i j}^{p}\right)^{-1},
$$

$\mu$ being the dynamic viscosity of the saturating fluid, while $K_{i j}^{f}$ and $K_{i j}^{p}$ are the permeability tensors in the macro and microphases. The term $\zeta_{i j}$ is a symmetric tensor representing the momentum transfer term but allowing for an anisotropic nature.

The boundary conditions imposed are that

$$
U_{i}^{f}=0, \quad U_{3}^{p}=0, \quad \text { on } z=0, d,
$$

and

$$
\left.\begin{array}{l}
\alpha_{L}\left(\frac{\partial T}{\partial z}+\beta\right) d-\left(1-\alpha_{L}\right) T=-\left(1-\alpha_{L}\right) T_{L}, \quad z=0, \\
\alpha_{U}\left(\frac{\partial T}{\partial z}+\beta\right) d+\left(1-\alpha_{U}\right) T=\left(1-\alpha_{U}\right) T_{U}, \quad z=d,
\end{array}\right\}
$$

where $T_{L}>T_{U}>0$ are constants and $0 \leqslant \alpha_{L}<1,0 \leqslant \alpha_{U}<1$, are likewise constants. The parameter $\beta=\left(T_{L}-T_{U}\right) / d$. In $\S 6$, where we present numerical results, we restrict our attention to $\alpha_{L}=0=\alpha_{U}$, i.e. prescribed temperatures on $z=0$ and $d$. However, for our general result on nonlinear stability it is expedient to allow the more general boundary conditions. The $\partial T / \partial z$ term is important when radiation boundary effects are acting, such as solar heating. Prescribed temperatures on the boundaries are likely under laboratory conditions or under cloudy skies, and (3.3) encompass the general situation since $\alpha_{L}$ and $\alpha_{U}$ may be taken in the range $[0,1)$. 
In order to investigate thermal convection we observe that (3.1), (3.2) and (3.3) admit the steady (conduction) solution

$$
\bar{U}_{i}^{f} \equiv 0, \quad \bar{U}_{i}^{p} \equiv 0, \quad \bar{T}=T_{L}-\beta z .
$$

To analyse the stability of solution (3.4) we let $u_{i}^{f}, u_{i}^{p}, \theta, \pi^{f}, \pi^{p}$ be perturbations to the steady solution. We then non-dimensionalize the resulting perturbation equations with the scalings

$$
x_{i}=x_{i}^{*} d, \quad t=t^{*} \mathcal{T}, \quad \mathcal{T}=\frac{(\rho c)_{m} d^{2}}{\kappa_{m}}, \quad U=\frac{\kappa_{m}}{(\rho c)_{f} d}, \quad P=d m_{11} U,
$$

where $\mathcal{T}, U$ and $P$ are time, velocity and pressure scales. The number $m_{11}$ is given by $m_{11}=M_{11}^{f}$ and we take this out as a factor in $M_{i j}^{f}$. We also write $M_{11}^{p}=\omega m_{11}$ and rescale $M_{i j}^{p}$. Then put $\lambda_{i j}=\zeta_{i j} / m_{11}, \lambda=\tilde{\mu} / d^{2} m_{11}$, define the temperature scale as

$$
T^{\sharp}=\mathrm{d} U \sqrt{\frac{(\rho c)_{f} \beta m_{11}}{\rho_{F} \alpha g \kappa_{m}}}
$$

and define the Rayleigh number $R a=R^{2}$ by

$$
R a=\frac{\rho_{F} \alpha g \beta d^{2}}{m_{11}\left[\kappa_{m} /(\rho c)_{f}\right]} .
$$

The non-dimensionalization allows us to reduce the number of parameters in (3.1). For example, in the isotropic case there are in (2.3) the parameters $\mu, \tilde{\mu}, K_{f}, K_{p}, \zeta, \rho_{F}, g$, $\alpha,(\rho c)_{m},(\rho c)_{f}$ and $\kappa_{m}$ and the non-dimensionalization reduces this to four parameters, a Rayleigh number, and non-dimensional versions of $\lambda, \zeta$ and $K_{r}=K_{f} / K_{p}$ the relative permeability, see $\S 6$. We are able to reduce the behaviour of thermal convection in that case to a study of the dependence of the Rayleigh number, $R a$, and the wavenumber upon the three parameters $\lambda, \zeta$ and $K_{r}$.

The resulting perturbation equations arising from (3.1) may be written as

$$
\begin{gathered}
\lambda \Delta u_{i}^{f}-M_{i j}^{f} u_{j}^{f}-\lambda_{i j}\left(u_{i}^{f}-u_{i}^{p}\right)-\pi_{, i}^{f}+R k_{i} \theta=0, \\
u_{i, i}^{f}=0, \\
-\omega M_{i j}^{p} u_{j}^{p}+\lambda_{i j}\left(u_{i}^{f}-u_{i}^{p}\right)-\pi_{, i}^{p}+R k_{i} \theta=0, \\
u_{i, i}^{p}=0, \\
\theta_{, t}+\left(u_{i}^{f}+u_{i}^{p}\right) \theta_{, i}=R\left(w^{f}+w^{p}\right)+\Delta \theta,
\end{gathered}
$$

where $\boldsymbol{u}^{f}=\left(u^{f}, v^{f}, w^{f}\right)$ and $\boldsymbol{u}^{p}=\left(u^{p}, v^{p}, w^{p}\right)$. These equations hold in the domain $(x, y) \in \mathbb{R}^{2},\{z \in(0,1)\}, t>0$. The boundary conditions are

$$
\left.\begin{array}{rl}
u_{i}^{f}=0, \quad w^{p} & =0, \quad z=0,1, \\
\theta_{z}-L_{L} \theta & =0, \quad z=0, \\
\theta_{z}+L_{U} \theta & =0, \quad z=1,
\end{array}\right\}
$$

where $L_{L}=\left(1-\alpha_{L}\right) / \alpha_{L}$ and $L_{U}=\left(1-\alpha_{U}\right) / \alpha_{U}$. In addition, we suppose $u_{i}^{f}, u_{i}^{p}, \theta, \pi^{f}$, $\pi^{p}$ satisfy a plane tiling shape in the $(x, y)$ plane. The periodic convection cell which arises will be denoted by $V$. 


\section{Instability and exchange of stabilities}

To determine the linear instability boundary we discard the nonlinear terms in (3.6) and seek a solution in which $u_{i}^{f}, u_{i}^{p}, \theta, \pi^{f}, \pi^{f}$ have time dependence like $e^{\sigma t}$. The system which remains is written as

$$
\left.\begin{array}{c}
\lambda \Delta u_{i}^{f}-M_{i j}^{f} u_{j}^{f}-\lambda_{i j}\left(u_{j}^{f}-u_{j}^{p}\right)-\pi_{, i}^{f}+R k_{i} \theta=0, \\
u_{i, i}^{f}=0, \\
-\omega M_{i j}^{p} u_{j}^{p}+\lambda_{i j}\left(u_{j}^{f}-u_{j}^{p}\right)-\pi_{, i}^{p}+R k_{i} \theta=0, \\
u_{i, i}^{p}=0, \\
\sigma \theta=R\left(w^{f}+w^{p}\right)+\Delta \theta .
\end{array}\right\}
$$

The boundary conditions are (3.7), together with periodicity in $(x, y)$. We now establish the strong form of the principle of exchange of stabilities. To achieve this, multiply equation $(4.1)_{1}$ by $u_{i}^{f *}$, the complex conjugate of $u_{i}^{f}$, and integrate over the period cell $V$. Next, multiply $(4.1)_{2}$ by $u_{i}^{p *}$ and multiply $(4.1)_{3}$ by $\theta^{*}$ and likewise integrate each resulting equation over $V$. Denote by $\langle\cdot\rangle$ integration over $V$, and let $\Gamma_{1}$ be the boundary of $V$ which intersects the plane $z=0$ while $\Gamma_{2}$ is the boundary of $V$ which intersects the plane $z=1$. Upon performing some integration by parts and using the boundary conditions one may derive the equations

$$
\left.\begin{array}{c}
-\lambda\left\langle u_{i, j}^{f} u_{i, j}^{f *}\right\rangle-\left\langle M_{i j}^{f} u_{j}^{f} u_{i}^{f *}\right\rangle \\
-\left\langle\lambda_{i j}\left(u_{j}^{f}-u_{j}^{p}\right) u_{i}^{f *}\right\rangle+R\left\langle\theta w^{f *}\right\rangle=0, \\
-\omega\left\langle M_{i j}^{p} u_{j}^{p} u_{i}^{p *}\right\rangle-\left\langle\lambda_{i j}\left(u_{j}^{p}-u_{j}^{f}\right) u_{i}^{p *}\right\rangle+R\left\langle\theta w^{p *}\right\rangle=0, \\
\sigma\left\langle\theta \theta^{*}\right\rangle=R\left[\left\langle w^{f} \theta^{*}\right\rangle+\left\langle w^{p} \theta^{*}\right\rangle\right]-\left\langle\theta_{, i} \theta_{, i}^{*}\right\rangle \\
+\int_{\Gamma_{2}} \theta^{*} \frac{\partial \theta}{\partial z} \mathrm{~d} A-\int_{\Gamma_{1}} \theta^{*} \frac{\partial \theta}{\partial z} \mathrm{~d} A .
\end{array}\right\}
$$

Next, add the above three equations and use the boundary conditions to obtain

$$
\left.\begin{array}{c}
\sigma\left\langle\theta \theta^{*}\right\rangle=-\lambda\left\langle u_{i, j}^{f} u_{i, j}^{f *}\right\rangle-\left\langle M_{i j}^{f} u_{j}^{f} u_{i}^{f *}\right\rangle \\
-\omega\left\langle M_{i j}^{p} u_{j}^{p} u_{i}^{p *}\right\rangle-\left\langle\lambda_{i j}\left(u_{j}^{f}-u_{j}^{p}\right)\left(u_{i}^{f *}-u_{i}^{p *}\right)\right\rangle \\
+R\left[\left\langle\theta w^{f *}\right\rangle+\left\langle w^{f} \theta^{*}\right\rangle+\left\langle\theta w^{p *}\right\rangle+\left\langle w^{p} \theta^{*}\right\rangle\right] \\
-\left\langle\theta_{, i} \theta_{, i}^{*}\right\rangle-L_{L} \int_{\Gamma_{1}} \theta^{*} \theta \mathrm{d} A-L_{U} \int_{\Gamma_{2}} \theta^{*} \theta \mathrm{d} A .
\end{array}\right\}
$$

One now writes each of $u_{i}^{f}, u_{i}^{p}, \theta$ as a sum of their real and imaginary parts and we put $\sigma=\sigma_{r}+i \sigma_{1}$. The imaginary part of equation (4.3) yields the result

$$
\sigma_{1}\left\langle\theta \theta^{*}\right\rangle=0
$$

One requires $\left\langle\theta \theta^{*}\right\rangle \neq 0$ and so $\sigma_{1}=0$. Thus, $\sigma \in \mathbb{R}$ and hence oscillatory convection does not occur. In conclusion, the strong form of the principle of exchange of stabilities is demonstrated. To find the linear instability boundary one may now investigate equations (4.1) with $\sigma=0$. 
We stress that in the two temperature case of Nield \& Kuznetsov (2006a) the exchange of stabilities has not been proved.

We return to calculate the instability threshold explicitly in $\S 6$. There, we restrict attention to the case where $\alpha_{U}=0, \alpha_{L}=0$, i.e. prescribed lower temperature $T_{L}$, upper temperature $T_{U}$. Detailed numerical results are presented.

\section{Global nonlinear stability}

Up to this point we have discussed only linear instability. We now establish an important result for global nonlinear stability (i.e. for all initial data). To achieve this result we employ the full nonlinear equations (3.6). We begin by multiplying (3.6) 1 by $u_{i}^{f}$ and integrating over $V$. Then, we multiply (3.6) $)_{3}$ by $u_{i}^{p}$ and integrate over $V$, and finally we multiply (3.6) $)_{5}$ by $\theta$ and integrate over $V$. We add the results for the first two resulting equations and then after some integration by parts and use of the boundary conditions we obtain the following two results

$$
\left.\begin{array}{c}
-\lambda\left\langle u_{i, j}^{f} u_{i, j}^{f}\right\rangle-\left\langle M_{i j}^{f} u_{j}^{f} u_{i}^{f}\right\rangle-\omega\left\langle M_{i j}^{p} u_{j}^{p} u_{i}^{p}\right\rangle \\
-\left\langle\lambda_{i j}\left(u_{j}^{f}-u_{j}^{p}\right)\left(u_{i}^{f}-u_{i}^{p}\right)\right\rangle+R\left\langle\theta\left(w^{f}+w^{p}\right)\right\rangle=0, \\
\frac{\mathrm{d}}{\mathrm{d} t} \frac{1}{2}\left\langle\theta^{2}\right\rangle=R\left\langle\theta\left(w^{f}+w^{p}\right)\right\rangle-\left\langle\theta_{, i} \theta_{, i}\right\rangle \\
-L_{L} \int_{\Gamma_{1}} \theta^{2} \mathrm{~d} A-L_{U} \int_{\Gamma_{2}} \theta^{2} \mathrm{~d} A .
\end{array}\right\}
$$

Add the equations in (5.1) to obtain

$$
\frac{\mathrm{d}}{\mathrm{d} t} \frac{1}{2}\left\langle\theta^{2}\right\rangle=R I-D,
$$

where now the production term $I$ is

$$
I=2\left\langle\left(w^{f}+w^{p}\right) \theta\right\rangle,
$$

and the dissipation term $D$ is given by

$$
\left.\begin{array}{rl}
D= & \lambda\left\langle u_{i, j}^{f} u_{i, j}^{f}\right\rangle+\left\langle M_{i j}^{f} u_{i}^{f} u_{j}^{f}\right\rangle+\omega\left\langle M_{i j}^{p} u_{i}^{p} u_{j}^{p}\right\rangle \\
& +\left\langle\lambda_{i j}\left(u_{j}^{f}-u_{j}^{p}\right)\left(u_{i}^{f}-u_{i}^{p}\right)\right\rangle+\left\langle\theta_{, i} \theta_{, i}\right\rangle \\
& +L_{U} \int_{\Gamma_{2}} \theta^{2} \mathrm{~d} A+L_{L} \int_{\Gamma_{1}} \theta^{2} \mathrm{~d} A .
\end{array}\right\}
$$

Define the threshold $R_{E}$ by

$$
\frac{1}{R_{E}}=\max _{H} \frac{I}{D}
$$

where $H$ is the space of admissible functions. From the energy identity (5.2) we may see that

$$
\frac{\mathrm{d}}{\mathrm{d} t} \frac{1}{2}\left\langle\theta^{2}\right\rangle \leqslant-D\left(1-\frac{R}{R_{E}}\right) .
$$

If now $R<R_{E}$, say $1-R / R_{E}=b>0$, then by using Poincaré's inequality in (5.6) one may obtain

$$
\frac{\mathrm{d}}{\mathrm{d} t} \frac{1}{2}\left\langle\theta^{2}\right\rangle \leqslant-b \pi^{2}\left\langle\theta^{2}\right\rangle .
$$


This may be integrated to see that

$$
\left\langle\theta(t)^{2}\right\rangle \leqslant\left\langle\theta(0)^{2}\right\rangle \exp \left(-2 b \pi^{2} t\right) .
$$

From inequality (5.8) one deduces that $\left\langle\theta(t)^{2}\right\rangle$ decays exponentially provided $R<R_{E}$.

To also establish decay of $u_{i}^{f}$ and $u_{i}^{p}$ we require $M_{i j}^{f}$ and $M_{i j}^{p}$ to be positive-definite and $\lambda_{i j}$ to be non-negative. These are realistic physical assumptions. If

$$
M_{i j}^{f} \xi_{i} \xi_{j} \geqslant k^{f} \xi_{i} \xi_{i}, \quad \text { and } \quad M_{i j}^{p} \xi_{i} \xi_{j} \geqslant k^{p} \xi_{i} \xi_{i}, \quad \text { for all } \xi_{i},
$$

$k^{f}>0, k^{p}>0$, then from equation $(5.1)_{1}$ we may derive using the arithmetic-geometric mean inequality

$$
\begin{gathered}
k^{f}\left\langle u_{i}^{f} u_{i}^{f}\right\rangle+k^{p} \omega\left\langle u_{i}^{p} u_{i}^{p}\right\rangle+\lambda\left\langle u_{i, j}^{f} u_{i, j}^{f}\right\rangle \leqslant \frac{R \gamma_{1}}{2}\left\langle w_{f}^{2}\right\rangle \\
+\frac{R \gamma_{2}}{2}\left\langle w_{p}^{2}\right\rangle+\frac{R}{2}\left(\frac{1}{\gamma_{1}}+\frac{1}{\gamma_{2}}\right)\left\langle\theta^{2}\right\rangle,
\end{gathered}
$$

for constants $\gamma_{1}>0, \gamma_{2}>0$. Choose $\gamma_{1}=k^{f} / R$ and $\gamma_{2}=k^{p} \omega / R$. Then (5.9) allows us to derive

$$
k^{f}\left\langle u_{i}^{f} u_{i}^{f}\right\rangle+k^{p} \omega\left\langle u_{i}^{p} u_{i}^{p}\right\rangle+2 \lambda\left\langle u_{i, j}^{f} u_{i, j}^{f}\right\rangle \leqslant K\left\langle\theta^{2}\right\rangle,
$$

where $K=R^{2}\left(1 / k^{f}+1 / k^{p} \omega\right)$. Since (5.8) shows $\left\langle\theta^{2}\right\rangle$ decays exponentially in time we may deduce $R<R_{E}$ is sufficient also for decay of $u_{i}^{f}$ and $u_{i}^{p}$ as shown in (5.10).

Hence, the number $R_{E}$ represents a global nonlinear stability threshold. We wish to compare this number to the analogous one of linear instability. To do this we derive the Euler-Lagrange equations for the maximum in (5.5). This follows a standard procedure whereby one replaces $u_{i}^{f}$ by $u_{i}^{f}+\epsilon \eta_{i}^{f}, u_{i}^{p}$ by $u_{i}^{p}+\epsilon \eta_{i}^{p}$, and $\theta$ by $\theta+\epsilon \eta$ and then one differentiates $I / D$ with respect to $\epsilon$ and takes the limit $\epsilon \rightarrow 0$. Upon completing this procedure we find for Lagrange multipliers $\omega^{f}$ and $\omega^{p}$ the Euler-Lagrange equations are

$$
\left.\begin{array}{c}
\lambda \Delta u_{i}^{f}-M_{i j}^{f} u_{j}^{f}-\lambda_{i j}\left(u_{j}^{f}-u_{j}^{p}\right)+R_{E} k_{i} \theta=\omega_{, i}^{f}, \\
u_{i, i}^{f}=0, \\
-\omega M_{i j}^{p} u_{j}^{p}+\lambda_{i j}\left(u_{j}^{f}-u_{j}^{p}\right)+R_{E} k_{i} \theta=\omega_{, i}^{p}, \\
u_{i, i}^{p}=0, \\
R_{E}\left(w^{f}+w^{p}\right)+\Delta \theta=0,
\end{array}\right\}
$$

together with the boundary conditions

$$
u_{i}^{f}=0, \quad w^{p}=0, \quad z=0,1,
$$

and

$$
\left.\begin{array}{l}
\theta_{z}+L_{U} \theta=0, \quad z=1, \\
\theta_{z}-L_{L} \theta=0, \quad z=0 .
\end{array}\right\}
$$

Since exchange of stabilities holds we may take $\sigma=0$ in (4.1) and we see that (5.11)-(5.13) are exactly the same as (3.7)-(4.1) for the linear instability threshold. 
Thus, we have shown that the linear instability problem yields the same Rayleigh number threshold as the fully nonlinear stability one. This is an optimal result which shows that the linear theory is capturing correctly the physics for the onset of convective motion. In $\S 8$ we find the linear instability thresholds and we know these also represent the nonlinear stability ones.

We again stress that in the two temperature case exchange of stabilities has not been proved. Straughan $(2009,2015)$ analyses linear instability and nonlinear energy stability results in detail for the two temperature situation where Brinkman theory is employed in both macro and micropores, and in the case where Darcy theory is employed in both. In the case of the Brinkman theory the energy stability limit is lower than the linear instability one. For the Darcy case the energy limit is much lower. This does suggest that there may well be oscillatory instability in the Darcy case. We should point out that the equivalence of linear instability and nonlinear stability proved in the present situation is a strong result which is not to be expected in general, see Xiong \& Chen (2019).

\section{Heated below isotropic theory, two free surfaces}

In this section we solve the linear instability problem for (4.1). We restrict attention to the case of isotropic permeability tensors and so $M_{i j}^{f} \equiv\left(\mu / K_{f}\right) \delta_{i j}$ and $M_{i j}^{p} \equiv\left(\mu / K_{p}\right) \delta_{i j}$ where $K_{f}$ and $K_{p}$ are the values of the permeability in the macro and microphases. We also suppose the interaction term is isotropic and so $\lambda_{i j} \equiv \xi \delta_{i j}$, where $\xi \geqslant 0$, is the non-dimensional interaction coefficient. We also restrict our attention to the case where $\alpha_{L}$ and $\alpha_{U}$ are zero so that the boundary conditions on the temperature are that

$$
T=T_{L}, \quad z=0 ; \quad T=T_{U}, \quad z=d,
$$

in the original problem.

Thus, the relevant system of equations becomes

$$
\left.\begin{array}{c}
\lambda \Delta u_{i}^{f}-u_{i}^{f}-\xi\left(u_{i}^{f}-u_{i}^{p}\right)-\pi_{, i}^{f}+R k_{i} \theta=0, \\
u_{i, i}^{f}=0, \\
-K_{r} u_{i}^{p}-\xi\left(u_{i}^{p}-u_{i}^{f}\right)-\pi_{, i}^{p}+R k_{i} \theta=0, \\
u_{i, i}^{p}=0, \\
R\left(w^{f}+w^{p}\right)+\Delta \theta=0,
\end{array}\right\}
$$

where the Rayleigh number is now given by

$$
R a=R^{2}=\frac{\beta d^{2} \rho_{F} g \alpha K_{f}}{\mu k},
$$

where $k=\kappa_{m} /(\rho c)_{f}$. The key parameters in (6.1) are $\lambda, \xi$ and $K_{r}$ and these are defined by

$$
\lambda=\frac{\tilde{\mu} K_{f}}{d^{2} \mu}, \quad \xi=\frac{\zeta K_{f}}{\mu}, \quad K_{r}=\frac{K_{f}}{K_{p}} .
$$

The coefficient $\lambda$ is essentially a non-dimensional measure of the Brinkman viscosity coefficient, or alternatively may be thought of as a non-dimensional version of the ratio of the Brinkman viscosity to the dynamic viscosity of the saturating fluid. 
The parameter $\xi$ is a non-dimensional version of the coefficient of momentum transfer between the macro and microphases. The coefficient $K_{r}$ is the ratio of the macropermeability to the micropermeability.

The boundary conditions on the perturbation variables are

$$
u_{i}^{f}=0, \quad w^{p}=0, \quad \theta=0, \quad z=0,1
$$

Due to the presence of the Brinkman term we require a further boundary condition on $w^{f}$. This depends on whether we treat stress free surfaces or fixed surfaces. The fixed surface problem, which involves more intricate numerical computation of eigenvalues of a singular problem, is addressed in $\$ 7$.

Next, take curlcurl of $(6.1)_{1,3}$ and retain the third components to derive the system of equations

$$
\left.\begin{array}{c}
(1+\xi) \Delta w^{f}-\xi \Delta w^{p}-R \Delta^{*} \theta-\lambda \Delta^{2} w^{f}=0, \\
\left(K_{r}+\xi\right) \Delta w^{p}-\xi \Delta w^{f}-R \Delta^{*} \theta=0, \\
R\left(w^{f}+w^{p}\right)+\Delta \theta=0,
\end{array}\right\}
$$

where $\Delta^{*}=\partial^{2} / \partial x^{2}+\partial^{2} / \partial y^{2}$. We seek a solution to these equations of form $w^{f}=$ $W^{f}(z) h(x, y)$ where $h$ is a planform which satisfies $\Delta^{*} h=-a^{2} h$, cf. Chandrasekhar (1981, pp. 43-52), where $a$ is the wavenumber, with a similar representation for $w^{p}$ and $\theta$. The functions $W^{f}, W^{p}$ and $\Theta$ are composed of terms like $\sin n \pi z, n=1,2, \ldots$ This results in (6.5) leading to an expression for $R^{2}$ in terms of $\Lambda_{n}=n^{2} \pi^{2}+a^{2}$. One may differentiate this equation in $n^{2}$ and conclude $n=1$ yields the minimum value and then the critical Rayleigh number is found by minimizing

$$
R^{2}=\frac{\lambda\left(K_{r}+\xi\right) \Lambda^{3}+\left(K_{r}+K_{r} \xi+\xi\right) \Lambda^{2}}{a^{2}\left(\lambda \Lambda+4 \xi+K_{r}+1\right)},
$$

in $a$, where $\Lambda=\pi^{2}+a^{2}$. Results are presented below for minimization of $R^{2}$ to yield the critical values of $R a$ and $a$, in terms of $\xi, K_{r}$ and $\lambda$.

Two limit cases which arise from (6.6) are worth noting. We observe that as $\lambda \rightarrow 0$ the critical value of $R a$ is found as

$$
R a=\left(\frac{K_{r}+K_{r} \xi+\xi}{1+4 \xi+K_{r}}\right) 4 \pi^{2},
$$

as obtained in Gentile \& Straughan (2017a). Furthermore, in the formal limit $K_{r} \rightarrow \infty$, one finds

$$
R^{2} \sim \frac{\lambda \Lambda^{3}+(1+\xi) \Lambda^{2}}{a^{2}},
$$

and then as $\xi \rightarrow 0$ we obtain the single-porosity case analysed by Rees (2002). For the Brinkman-Darcy theory studied here we find experimental values of $K_{f}$ and $K_{r}$ which suggest $K_{r} \gg 1$.

To understand the behaviour of the critical Rayleigh number in both free and fixed surface cases it is helpful to recollect the energy equation (5.2) and the forms for $I$ and $D$ in (5.3) and (5.4). In the heated from below isotropic case under analysis in this section the function $I$ is as defined in (5.3). Note that none of the parameters $\lambda, K_{r}, \xi$ appear in $I$ but they are all in $D$, which here has the form 


$$
\begin{aligned}
D= & \lambda\left\langle u_{i, j}^{f} u_{i, j}^{f}\right\rangle+\left\langle u_{i}^{f} u_{i}^{f}\right\rangle+K_{r}\left\langle u_{i}^{p} u_{i}^{p}\right\rangle \\
& +\xi\left\langle\left(u_{i}^{f}-u_{i}^{p}\right)\left(u_{i}^{f}-u_{i}^{p}\right)\right\rangle+\left\langle\theta_{, i} \theta_{, i}\right\rangle .
\end{aligned}
$$

Thus, we expect $\lambda, K_{r}$ and $\xi$ to each exert a stabilizing influence (in that the critical Rayleigh number increases with each parameter increasing) and this is exactly what we witness numerically for either free or fixed boundary conditions.

\section{Heated below isotropic theory, two fixed surfaces}

In this section we solve the linear instability problem for (6.1), but we now analyse the fixed surface problem. Thus, we solve equations (6.5) with normal modes and employ the boundary conditions

$$
w^{f}=0, \quad w^{f \prime}=0, \quad w^{p}=0, \quad \theta=0, \quad z=0,1,
$$

where $w^{f \prime}=\partial w^{f} / \partial z$. To solve this system numerically we use a modified $D^{2}$ Chebyshev tau method. The Chebyshev tau method is described in Orszag (1971), while the $D^{2}$ version is described in Dongarra, Straughan \& Walker (1996). Thus, we write, as before, $w^{f}=W^{f}(z) h(x, y)$, with a similar representation for $w^{p}$ and $\theta$. Let $D=\mathrm{d} / \mathrm{d} z$ and let $a$ be the wavenumber. Then (6.5) reduce to

$$
\left.\begin{array}{c}
\lambda\left(D^{2}-a^{2}\right)^{2} W^{f}-(1+\xi)\left(D^{2}-a^{2}\right) W^{p}-R a^{2} \Theta=0, \\
\left(K_{r}+\xi\right)\left(D^{2}-a^{2}\right) W^{p}-\xi\left(D^{2}-a^{2}\right) W^{f}+R a^{2} \Theta=0, \\
\left(D^{2}-a^{2}\right) \Theta+R\left(W^{f}+W^{p}\right)=0,
\end{array}\right\}
$$

for $0<z<1$, which are to be solved together with the boundary conditions

$$
W^{f}=0, \quad D W^{f}=0, \quad W^{p}=0, \quad \Theta=0, \quad z=0,1 .
$$

The modified method we employ introduces variables $\chi$ and $\psi$ by

$$
\chi=\left(D^{2}-a^{2}\right) W^{f} \quad \text { and } \quad \psi=\left(D^{2}-a^{2}\right) W^{p} .
$$

Equations (7.2) are then rewritten as the system

$$
\begin{gathered}
\left(D^{2}-a^{2}\right) W^{f}-\chi=0, \\
\left(D^{2}-a^{2}-\frac{1+\xi}{\lambda}\right) \chi+\frac{\xi}{\lambda} \psi=\frac{R}{\lambda} a^{2} \Theta, \\
\left(D^{2}-a^{2}\right) W^{p}-\psi=0, \\
\psi-\left(\frac{\xi}{K_{r}+\xi}\right) \chi=-\frac{R}{\left(K_{r}+\xi\right)} a^{2} \Theta, \\
\left(D^{2}-a^{2}\right) \Theta=-R\left(W^{f}+W^{p}\right) .
\end{gathered}
$$

In terms of the Chebyshev polynomials $T_{n}(z)$ we now write 


$$
\begin{gathered}
W^{f}=\sum_{n=0}^{N} W_{n}^{f} T_{n}(z), \quad \chi=\sum_{n=0}^{N} \chi_{n} T_{n}(z), \\
W^{p}=\sum_{n=0}^{N} W_{n}^{p} T_{n}(z), \quad \psi=\sum_{n=0}^{N} \psi_{n} T_{n}(z), \\
\Theta=\sum_{n=0}^{N} \Theta_{n} T_{n}(z) .
\end{gathered}
$$

In this way equations (7.4) reduce to solving the generalized eigenvalue problem

$$
\boldsymbol{A x}=R \boldsymbol{B x},
$$

where the matrices $\boldsymbol{A}$ and $\boldsymbol{B}$ are given by

$$
\boldsymbol{A}=\left(\begin{array}{ccccc}
D^{2}-a^{2} \boldsymbol{I} & -I & 0 & 0 & 0 \\
0 & D^{2}-\left(a^{2}+(1+\xi) \lambda^{-1}\right) I & 0 & (\xi / \lambda) I & 0 \\
0 & 0 & D^{2}-a^{2} I & -I & 0 \\
0 & -\left(\xi /\left(K_{r}+\xi\right)\right) I & 0 & I & 0 \\
0 & 0 & 0 & 0 & D^{2}-a^{2} I
\end{array}\right)
$$

and

$$
\boldsymbol{B}=\left(\begin{array}{ccccc}
0 & 0 & 0 & 0 & 0 \\
0 & 0 & 0 & 0 & \left(a^{2} / \lambda\right) I \\
0 & 0 & 0 & 0 & 0 \\
0 & 0 & 0 & 0 & -\left(a^{2} /\left(K_{r}+\xi\right)\right) I \\
-I & 0 & -I & 0 & 0
\end{array}\right)
$$

where $\boldsymbol{I}$ is the $n \times n$ identity matrix and 0 is the $n \times n$ zero matrix. The last two rows of each of the block matrices in rows $1,2,3$ and 5 contain the boundary conditions. Details of how to do this are given in Dongarra et al. (1996, pp. 406, 407). We make use of the fact that $T_{n}( \pm 1)=( \pm 1)^{n}$ and $T_{n}^{\prime}=( \pm 1)^{n+1} n^{2}$. The last two rows of blocks 1, 1 and 2, 1 of $A$ contain the boundary conditions for $W_{f}$ while the last two rows of block 3, 3 contains the boundary conditions for $W_{p}$. The last two rows of block 5 , 5 contains the boundary conditions for $\Theta$. The matrices in block rows 4 contain the complete $n \times n$ identity matrix for both $A$ and $B$.

The resulting finite dimensional generalized eigenvalue problem (7.5) is solved using the QZ algorithm of Moler \& Stewart (1971). We find this modified $D^{2}$ Chebyshev tau method works well and yields accurate results, although care has to be taken to avoid spurious eigenvalues.

\section{Numerical results}

We present numerical results for computations involving minimizing $R a$ in (6.6) in $a$ for two free surfaces and likewise minimizing in $a$ for the solution from (7.4) or (7.5) when the surfaces are fixed. In both cases the Rayleigh number is a function of the wavenumber, $a$, but also of the parameters $\lambda, \xi$ and $K_{r}$ defined in (6.3). We firstly assess what range of values these parameters may take in real bidisperse porous materials. In order to do this we make use of the articles of Givler \& Altobelli (1994), and of Hooman et al. (2015), and we employ the Carman-Kozeny relation for the permeability as given by Chen (1990) or Nield (2000). 
In their analysis of an open cell rigid foam Givler \& Altobelli (1994) deduce experimentally that

$$
5.1 \leqslant \frac{\tilde{\mu}}{\mu} \leqslant 10.9
$$

and they also calculate $K_{f}=3.3 \times 10^{-7} \mathrm{~m}^{2}$ with an error

$$
0.0027 .1 \leqslant K_{f} \leqslant 0.0042 \mathrm{~cm}^{2} .
$$

For the momentum transfer coefficient, $\zeta$, we have found only one source of information and this is Hooman et al. (2015) who report for a plate-fin heat exchanger

$$
\zeta=63.3 \mathrm{~Pa} \mathrm{~s} \mathrm{~m}^{-2} \text {. }
$$

Hooman et al. (2015) also supply values for the macro and micropermeabilities depending on the spacing between the plates and they report

$$
K_{f}=2.13 \times 10^{-7} \mathrm{~m}^{2},
$$

with (Hooman et al. (2015) table 2, different values corresponding to different spacing)

$$
K_{r}=\frac{K_{f}}{K_{p}}=701.75,456.14,263.16 .
$$

Observe that the value of $K_{f}$ in (8.4) is in keeping with those of Givler \& Altobelli (1994) in (8.2).

If we use the Carman-Kozeny relation of Chen (1990), then

$$
K_{f}=\frac{d_{f}^{2}}{172.8} \frac{\phi^{3}}{(1-\phi)^{2}},
$$

where $d_{f}$ is the diameter of the spheres comprising the porous medium and $\phi$ is the porosity. For a porosity of $\phi=0.8$ and $5 \mathrm{~mm}$ glass beads (8.6) yields a value for $K_{f}$ of

$$
K_{f}=1.85 \times 10^{-6} \mathrm{~m}^{2},
$$

which is consistent with (8.2) or (8.4).

If we use relation (8.6) to calculate $K_{r}$ then

$$
K_{r}=\frac{K_{f}}{K_{p}}=\frac{d_{f}^{2}}{d_{p}^{2}} \frac{\phi^{3}}{(1-\phi)^{2}} \frac{(1-\epsilon)^{2}}{\epsilon^{3}},
$$

where $d_{f}$ and $d_{p}$ denote the diameter of the spheres in the macro and microphases. With $\phi$ and $\epsilon$ the permeabilities, then for $d_{f} / d_{p}=10, \phi=0.8$ and $\epsilon=0.3$ we find $K_{r}=2322$.

We may also calculate the Brinkman parameter $\lambda$ using equation (8.6) then

$$
\lambda=\frac{\tilde{\mu}}{\mu} \frac{1}{172.8} \frac{d_{f}^{2}}{d^{2}} \frac{\phi^{3}}{(1-\phi)^{2}},
$$

where $d$ is the depth of the convection layer. For a layer depth of $3 \mathrm{~cm}$, with $3 \mathrm{~mm}$ glass beads, and assuming $\tilde{\mu} / \mu=7.5$ as in Givler \& Altobelli (1994), we find

$$
\lambda \approx 5.56 \times 10^{-3} .
$$


If we employ the Givler \& Altobelli (1994) of $K_{f}=3.3 \times 10^{-7} \mathrm{~m}^{2}$ then for a $3 \mathrm{~cm}$ layer we obtain

$$
\lambda \approx 2.75 \times 10^{-3} .
$$

If we take $d$ to be $1 \mathrm{~cm}$, take $K_{f}=4.2 \times 10^{-7}$ and $\tilde{\mu} / \mu=10.9$, which are in the range of the Givler \& Altobelli (1994) values, then we find

$$
\lambda=\frac{\tilde{\mu}}{\mu} \frac{K_{f}}{d^{2}} \approx 4.578 \times 10^{-2} .
$$

One may further employ equation (8.8) to see that if $d_{f} / d_{p}=5$ and we take $\phi=0.6$, $\epsilon=0.6$ then $K_{r}=25$ whereas for $d_{f} / d_{p}=4, \phi=0.8, \epsilon=0.6$ then $K_{r}=151.7$.

To calculate $\xi$ we use the relation

$$
\xi=\frac{\zeta K_{f}}{\mu}
$$

where $\xi$ is the momentum transfer coefficient which from Hooman et al. (2015) is given by (8.3). We take the fluid to be water at $25^{\circ} \mathrm{C}$ and then from the website engineersedge.com $\mu=8.9 \times 10^{-4} \mathrm{~Pa}$ s. We have derived above four typical values for the macropermeability, $K_{f}$, and these are

$$
K_{f}=1.85 \times 10^{-6} \mathrm{~m}^{2}, 2.13 \times 10^{-7} \mathrm{~m}^{2}, 3.3 \times 10^{-7} \mathrm{~m}^{2}, 4.2 \times 10^{-7} \mathrm{~m}^{2},
$$

which, using (8.9), lead to values of $\xi$ as

$$
\xi=1.515 \times 10^{-2}, 2.347 \times 10^{-2}, 2.987 \times 10^{-2}, 1.316 \times 10^{-1} .
$$

The range of $\lambda$ and $K_{r}$ values we have found are

$$
\left.\begin{array}{rl}
\lambda & =2.75 \times 10^{-3}, 5.56 \times 10^{-3}, 4.578 \times 10^{-2}, \\
K_{r} & =25,151.7,263.16,456.14,701.75,2322 .
\end{array}\right\}
$$

In our computations we mostly employ these values to determine the behaviour of the critical Rayleigh number, $R a$, and the critical wavenumber, $a$, as functions of the non-dimensional parameters $\lambda, \xi$ and $K_{r}$.

In both the fixed and free surface boundary condition cases the Rayleigh number is found to increase with variation in $K_{r}, \lambda$ or $\xi$. The variation in $R a$ with $K_{r}$ is shown in table 5 in the fixed case when $\lambda$ and $\xi$ take possible realistic values. To interpret these we recall the definition of $R a$ in (6.2). Since $K_{r}=K_{f} / K_{p}$ we may think of $K_{f}$ as fixed and then as $K_{r}$ increases $K_{p}$ decreases. This means the micropermeability decreases and so the fluid moves less easily in the micropores. In this case we expect convective motion to be less easy and so the system is more stable as is witnessed by increasing $R a$. The analogous variation in the critical value of the wavenumber, $a$ depends strongly on the boundary conditions. Since the wavenumber is inversely proportional to the cell width (aspect ratio) this means increasing $a$ leads to wider convection cells, whereas $a$ decreasing means narrower cells. In table 5 we see that, for fixed boundary conditions, $a$ increases as $K_{r}$ increases, although the increase in $a$ is not large. However, the free boundary case is very different, as seen in table 6 . When $\xi=0.1316$ and $\lambda=0.04578$ we find $a$ increases from the value 2.99266 when $K_{r}=0$ to a maximum of 2.99466 when $K_{r}=0.037$ and thereafter decreases to the 


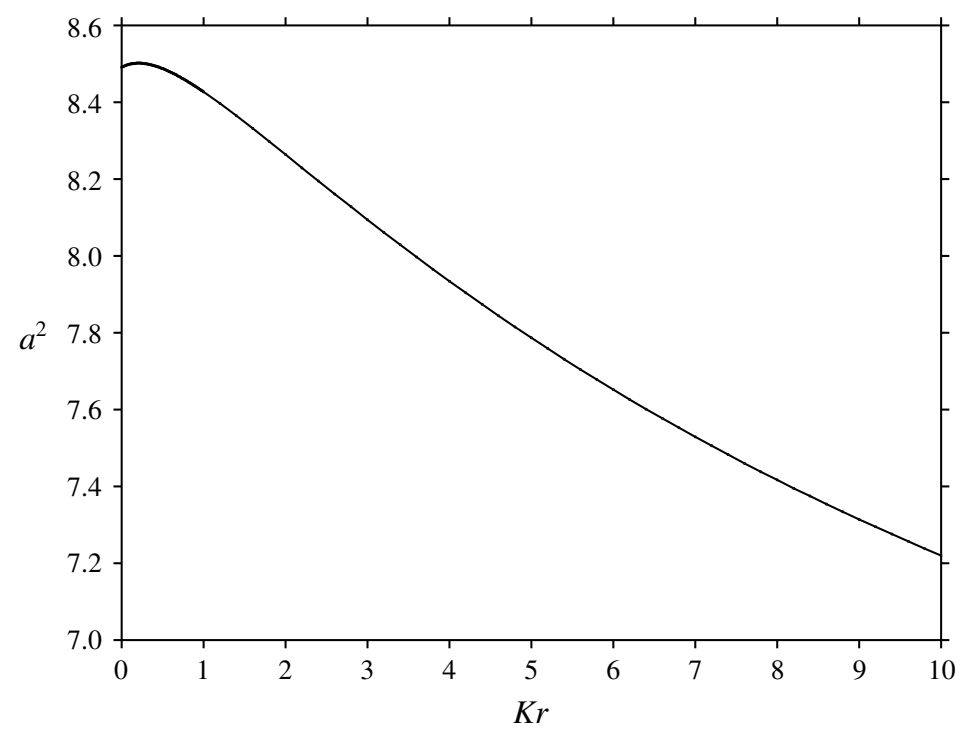

FIGURE 1. Graph of $a^{2}$ versus $K r$ with $K r$ varying in the range 0 to 10 , two free surfaces. Here, $\lambda=1$ and $\xi=1$. The values at the end points as shown are $\left(a^{2}, K r\right)=(8.491,0)$ and $\left(a^{2}, K r\right)=(7.220,10)$. The maximum value is approximately at $\left(a^{2}, K r\right)=(8.502,0.21)$.

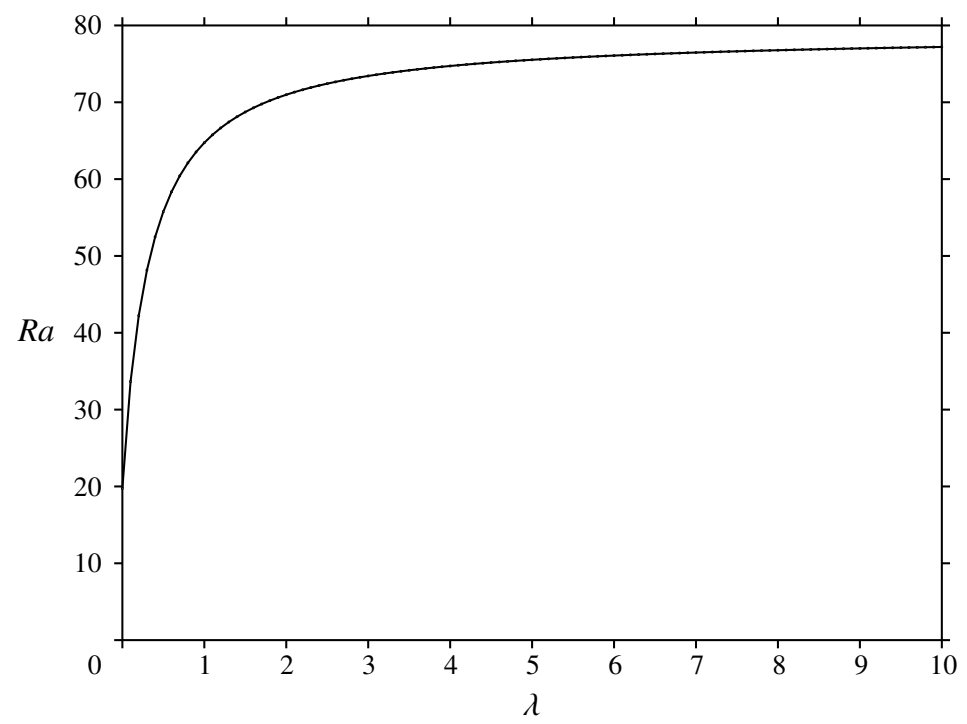

FIGURE 2. Graph of $R a$ versus $\lambda$ with $\lambda$ varying in the range 0 to 10 , two free surfaces. Here $K r=1$ and $\xi=1$. The values at the endpoints as shown are $(R a, \lambda)=(19.73921,0)$ and $(\operatorname{Ra} 2, \lambda)=(77.20063,10)$.

value $a=2.64953$ when $K_{r}=10^{4}$. For the case of $\xi=0.01515, \lambda=2.75 \times 10^{-3}$, $a$ decreases from the value 3.13720 at $K_{r}=0$ to $a=3.06643$ when $K_{r}=10^{4}$. The variation of $a^{2}$ against $K_{r}$ in the free case with $\lambda=1, \xi=1$ is shown in figure 3 . 


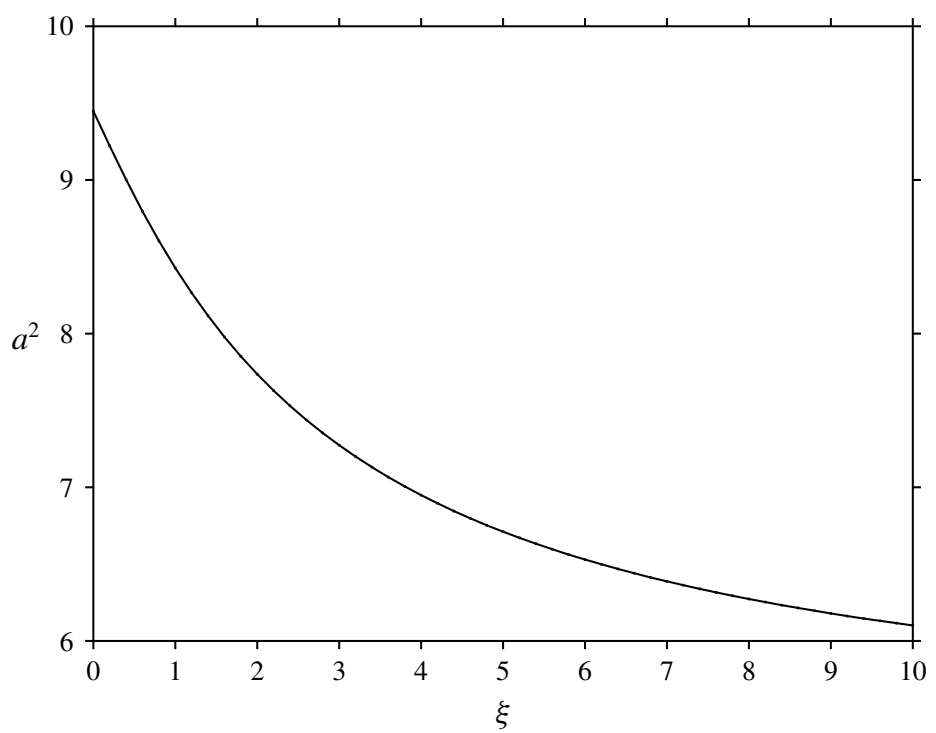

FIGURE 3. Graph of $a^{2}$ versus $\xi$ with $\xi$ varying in the range 0 to 10 , two free surfaces. Here $K r=1$ and $\lambda=1$. The values at the endpoints as shown are $\left(a^{2}, \xi\right)=(9.448,0)$ and $\left(a^{2}, \xi\right)=(6.101,10)$.

Figure 2 shows that $R a$ increases rapidly as the Brinkman coefficient $\lambda$ increases for $\lambda$ small, and then the growth is less rapid for larger $\lambda$. This is in agreement with the case of a single-porosity Brinkman material. The variation in the critical wavenumber $a$ in figures 4-6 is interesting. In our notation, in the single-porosity case replacing $w^{f}$ and $w^{p}$ by $w$, instead of (6.5) one has, cf. Rees (2002),

$$
\begin{gathered}
-\lambda \Delta^{2} w+\Delta w-R \Delta^{*} \theta=0, \\
\Delta \theta+R w=0
\end{gathered}
$$

and instead of (6.6) one finds

$$
R^{2}=\frac{\lambda \Lambda^{3}+\Lambda^{2}}{a^{2}} .
$$

(We stress that Rees (2002) analyses the fixed surface problem.) The presence of the $\lambda \Lambda$ term in the denominator of (6.6) changes the $a^{2}$ versus $\lambda$ behaviour significantly between the one porosity and the bidispersive situation. In the one-porosity case we find for two stress free surfaces that $a^{2}$ decreases from $\pi^{2}$ to $\pi^{2} / 2$ as $\lambda$ increases from 0 . In the one-porosity case the critical wavenumber may be obtained analytically and we find

$$
a_{c r}^{2}=\left\{-\left(\lambda \pi^{2}+1\right)+\left[\left(\lambda \pi^{2}+1\right)^{2}+8 \lambda \pi^{2}\left(1+\lambda \pi^{2}\right)\right]^{1 / 2}\right\} / 4 \lambda .
$$

Using Newton's binomial expansion we find for $\lambda$ small,

$$
a_{c r}^{2}=\pi^{2}-2 \pi^{4} \lambda+10 \pi^{6} \lambda^{2}+O\left(\lambda^{3}\right),
$$

which confirms the decrease for $\lambda \ll 1$. 


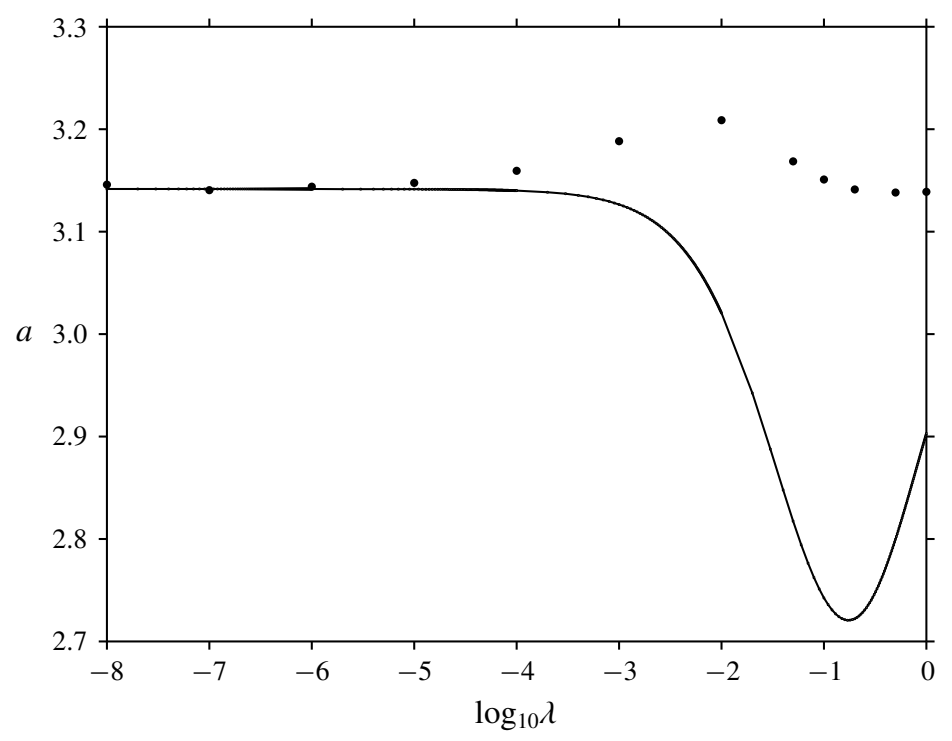

FIGURE 4. Graph of $a$ versus $\log _{10} \lambda$, for $\xi=1, K_{r}=1$. The solid curve indicates two free surfaces whereas the solid dots are for two fixed surfaces. The minimum on the solid curve is where $a=2.7207, \lambda=0.17$, and the maximum value displayed for fixed surfaces is $a=3.2088, \lambda=0.01$.

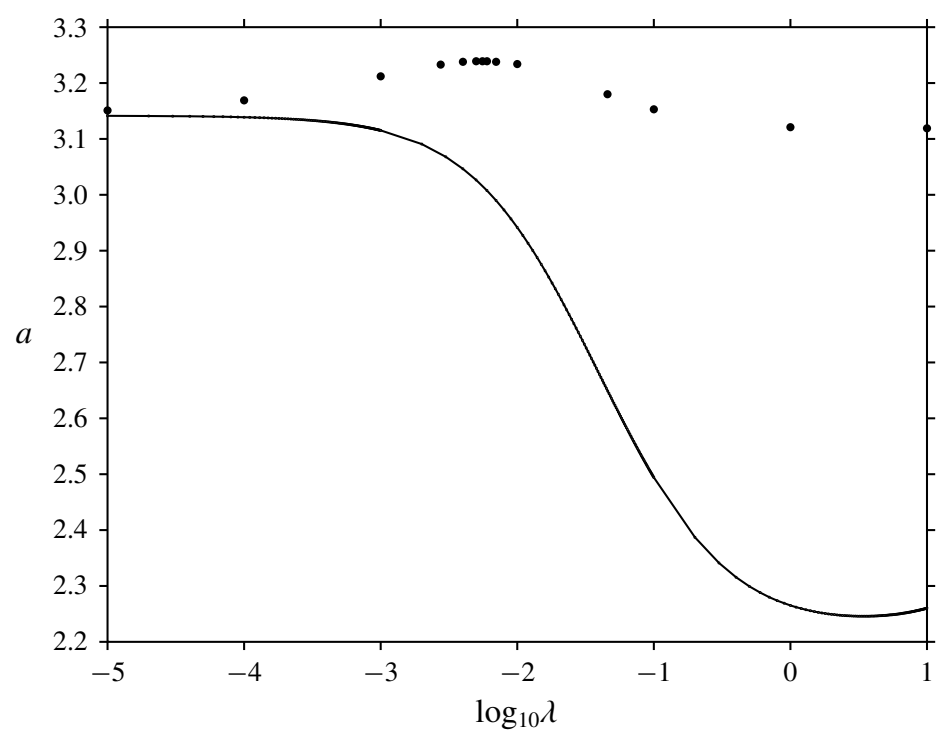

FIGURE 5. Graph of $a$ versus $\log _{10} \lambda$, for $\xi=0.1316, K_{r}=2322$. The solid curve indicates two free surfaces whereas the solid dots are for two fixed surfaces. The minimum on the solid curve is where $a=2.24586, \lambda=3.46$, and the maximum value displayed for fixed surfaces is $a=3.239, \lambda \in[0.005,0.006]$.

In the bidisperse case figures 4-6 show for two free surfaces a clear decrease then increase as $\lambda$ increases for small $\lambda$. The initial decrease may be verified by an asymptotic analysis from (6.6). If we form $\partial R^{2} / \partial a^{2}$ from (6.6) and equate to zero 


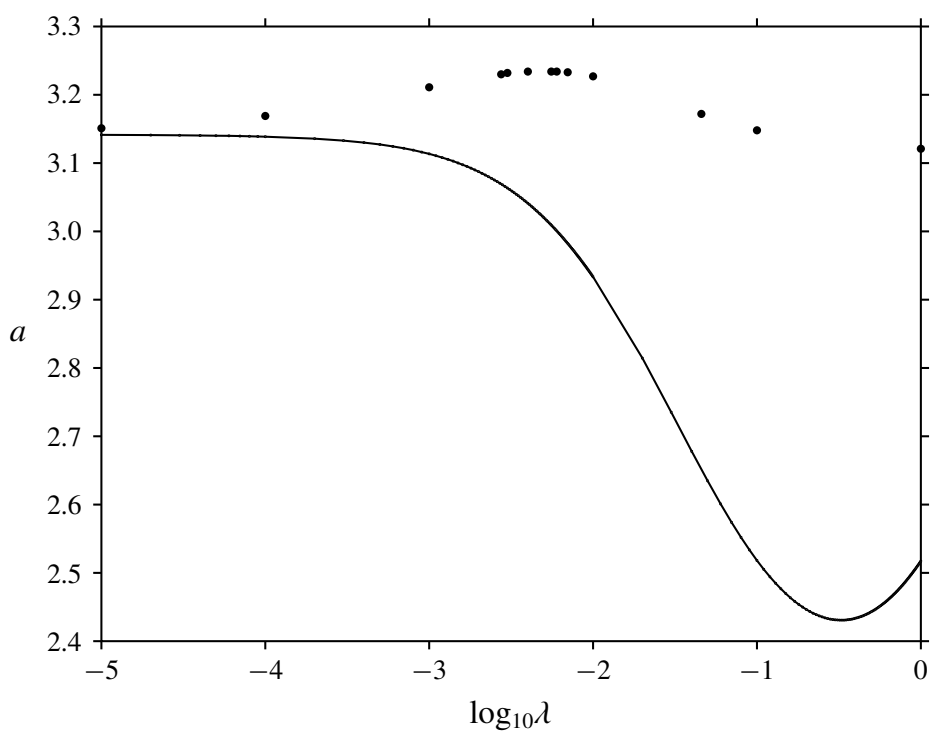

FIGURE 6. Graph of $a$ versus $\log _{10} \lambda$, for $\xi=0.01515, K_{r}=25$. The solid curve indicates two free surfaces whereas the solid dots are for two fixed surfaces. The minimum on the solid curve is where $a=2.43084, \lambda=0.33$, and the maximum value displayed for fixed surfaces is $a=3.234, \lambda \in[0.004,0.006]$.

and then solve the resulting equation by expanding $a^{2}$ as a power series in $\lambda$ then for $0<\lambda \ll 1$ we find

$$
a^{2}=\pi^{2}-X_{1} \lambda+O\left(\lambda^{2}\right), \quad \text { where } X_{1}=2 \pi^{6}\left[\frac{K_{r}^{2}+4 K_{r} \xi+4 \xi^{2}}{\left(K_{r}+K_{r} \xi+\xi\right)\left(1+K_{r}+4 \xi\right)}\right] .
$$

In fact, when $K_{r}=1, \xi=1$ the minimum value is $\left(a^{2}, \lambda\right)=(7.402,0.17)$ whereas for $K_{r}=5, \xi=0.01$ the minimum is at $\left(a^{2}, \lambda\right)=(6.953,0.15)$. Thus the presence of micropores is changing the behaviour of the cell shape. When micropores are present and $\lambda$ is small the cell shape increases in width (aspect ratio) as $\lambda$ increases, reaching a maximum and then decreasing again. The wavenumber variation for $\lambda$ small is, however, very different from the free-free case. We see that in tables 3 and 4 when the surfaces are fixed then the wavenumber has a maximum in $\lambda$. This is exactly the opposite behaviour to the free-free situation. This behaviour is, however, in agreement with the findings of Rees (2002) in the single-porosity case. He also predicts a rise then decrease in $a$ as $\lambda$ decreases. We have checked the behaviour of the critical value of $a$ in the free-free single-porosity case and it is as in figures 4-6. We have computed the critical Rayleigh number and wavenumber for various other combinations of possible realistic values of parameter values and we always see the same type of behaviour as $\lambda$ varies.

In figure 4 the maximum and minimum values for the fixed and free cases have reasonably close values of $\lambda$, but these are when $K_{r}=1, \xi=1$. For $K_{r}$ and $\xi$ values which we have calculated for possible real bidisperse materials figures 5 and 6 show that the maximum in $\lambda$ for the fixed surface problem is in the range of realistic values. The corresponding minima in $\lambda$ for the free surface situation are for larger values of $\lambda$.

The variations of $R a$ and $a$ in $\xi$ are given in tables 7 and 8 with potentially realistic values of parameters. For fixed surfaces the situation of the behaviour of $a$ is not clear. 


$\begin{array}{lcccc}\lambda & R a & a & R a & a \\ 10 & 78.1505 & 3.1412 & 195.5290 & 3.1411 \\ 5 & 77.3665 & 3.1408 & 193.333 & 3.1407 \\ 1 & 71.7965 & 3.1389 & 177.7278 & 3.1384 \\ 0.5 & 66.2211 & 3.1382 & 162.0961 & 3.1370 \\ 0.2 & 55.0540 & 3.1412 & 130.7612 & 3.1381 \\ 0.1 & 45.1771 & 3.1509 & 103.0351 & 3.1453 \\ 0.05 & 36.3571 & 3.1686 & 78.3048 & 3.1613 \\ 0.01 & 24.8655 & 3.2088 & 46.4013 & 3.2117 \\ 0.001 & 20.7784 & 3.1883 & 35.5830 & 3.2012 \\ 10^{-4} & 20.0082 & 3.1594 & 33.6974 & 3.1654 \\ 10^{-5} & 19.8181 & 3.1476 & 33.2537 & 3.1496 \\ 10^{-6} & 19.7626 & 3.1439 & 33.1260 & 3.1438 \\ 10^{-7} & 19.7510 & 3.1405 & 33.0962 & 3.1438 \\ 10^{-8} & 19.7495 & 3.1459 & 33.0923 & 3.1454\end{array}$

TABLE 2. Critical values of $R a$ and $a$ for quoted values of $\lambda$, two fixed surfaces. Columns 2 and 3 are for $K_{r}=1, \xi=1$, while columns 4 and 5 are for $K_{r}=5, \xi=0.01$.

$\begin{array}{lcccc}\lambda & R a & a & R a & a \\ 10 & 78.1505 & 3.1412 & 195.5290 & 3.1411 \\ 5 & 77.3665 & 3.1408 & 193.333 & 3.1407 \\ 1 & 71.7965 & 3.1389 & 177.7278 & 3.1384 \\ 0.5 & 66.2211 & 3.1382 & 162.0961 & 3.1370 \\ 0.2 & 55.0540 & 3.1412 & 130.7612 & 3.1381 \\ 0.1 & 45.1771 & 3.1509 & 103.0351 & 3.1453 \\ 0.05 & 36.3571 & 3.1686 & 78.3048 & 3.1613 \\ 0.01 & 24.8655 & 3.2088 & 46.4013 & 3.2117 \\ 0.001 & 20.7784 & 3.1883 & 35.5830 & 3.2012 \\ 10^{-4} & 20.0082 & 3.1594 & 33.6974 & 3.1654 \\ 10^{-5} & 19.8181 & 3.1476 & 33.2537 & 3.1496 \\ 10^{-6} & 19.7626 & 3.1439 & 33.1260 & 3.1438 \\ 10^{-7} & 19.7510 & 3.1405 & 33.0962 & 3.1438 \\ 10^{-8} & 19.7495 & 3.1459 & 33.0923 & 3.1454\end{array}$

TABLE 3. Critical values of $R a$ and $a$ for quoted values of $\lambda$, two fixed surfaces. Columns 2 and 3 are for $K_{r}=1, \xi=1$, while columns 4 and 5 are for $K_{r}=5, \xi=0.01$.

In table 7 , for $\lambda=4.578 \times 10^{-2}$ and $K_{r}=25$ or 2322 the critical wavenumber $a$ always increases as $\xi$ increases. However, from table 7 , for $\lambda=2.75 \times 10^{-3}$ and $K_{r}=25$ or 2322, $a$ always decreases. For the free surface case $a$ is found to increase in all four cases as seen in table 9 .

\section{Conclusions}

We have presented a model for thermal convection in a dual-porosity (bidisperse) porous medium which allows for the macropermeability to be relatively large compared to the micropermeability. The behaviour of the critical Rayleigh number, $R a$, and critical wavenumber, $a$, depends on three parameters, $\lambda, \xi$ and $K_{r}$ given in (6.3). We observe that these non-dimensional parameters represent the Brinkman coefficient, 


$\begin{array}{lcccc}\lambda & R a & a & R a & a \\ 10^{-5} & 44.923 & 3.151 & 38.710 & 3.151 \\ 10^{-4} & 45.598 & 3.169 & 39.306 & 3.169 \\ 10^{-3} & 48.468 & 3.212 & 41.861 & 3.211 \\ 2.75 \times 10^{-3} & 52.368 & 3.233 & 45.352 & 3.230 \\ 3.00 \times 10^{-3} & - & - & 45.809 & 3.232 \\ 4.00 \times 10^{-3} & 54.864 & 3.238 & 47.586 & 3.234 \\ 5.00 \times 10^{-3} & 56.785 & 3.239 & - & - \\ 5.560 \times 10^{-3} & 57.841 & 3.239 & 50.246 & 3.234 \\ 6.000 \times 10^{-3} & 58.664 & 3.239 & 50.979 & 3.234 \\ 7.000 \times 10^{-3} & 60.512 & 3.238 & 52.624 & 3.233 \\ 1.000 \times 10^{-2} & 65.942 & 3.234 & 57.433 & 3.227 \\ 4.578 \times 10^{-2} & 127.846 & 3.180 & 109.254 & 3.172 \\ 0.1 & 220.309 & 3.153 & 176.976 & 3.148 \\ 1 & 1724.917 & 3.121 & 1722.397 & 3.121 \\ 10 & 14438.318 & 3.119 & - & -\end{array}$

TABLE 4. Critical values of $R a$ and $a$ for quoted values of $\lambda$, two fixed surfaces. Columns 2 and 3 are for $K_{r}=2322, \xi=0.1316$, while columns 4 and 5 are for $K_{r}=25, \xi=0.01515$.

$\begin{array}{lcccc}K_{r} & R a & a & R a & a \\ 1 & 31.111 & 3.155 & 21.468 & 3.185 \\ 10 & 95.127 & 3.172 & 42.369 & 3.225 \\ 25 & 112.376 & 3.176 & 45.352 & 3.230 \\ 151.7 & 125.152 & 3.179 & 47.205 & 3.234 \\ 263.16 & 126.358 & 3.180 & 47.367 & 3.234 \\ 456.14 & 127.063 & 3.180 & 47.461 & 3.234 \\ 701.75 & 127.403 & 3.180 & 47.506 & 3.234 \\ 2322 & 127.846 & 3.180 & 47.564 & 3.235 \\ 10^{4} & 127.993 & 3.180 & 47.583 & 3.235\end{array}$

TABLE 5. Critical values of $R a$ and $a$ for quoted values of $K r$, two fixed surfaces. Columns 2 and 3 are for $\lambda=0.04578, \xi=0.1316$, while columns 4 and 5 are for $\lambda=0.00275, \xi=0.01515$.

$\begin{array}{lcc}K_{r} & a & a \\ 0 & 2.9927 & 3.1372 \\ 10 & 2.7072 & 3.0733 \\ 100 & 2.6561 & 3.0672 \\ 500 & 2.6508 & 3.0666 \\ 1000 & 2.6503 & 3.0666 \\ 5000 & 2.6497 & 3.0664 \\ 10^{4} & 2.6495 & 3.0664\end{array}$

TABLE 6. Critical values of the wavenumber $a$ for quoted values of $K r$, two free surfaces. Columns 2 and 3 are for $\lambda=0.04578, \xi=0.1316$, while columns 4 and 5 are for $\lambda=$ $0.00275, \xi=0.01515$.

the mass transfer coefficient between the macro and microphases and $K_{r}=K_{f} / K_{p}$ represents the ratio of macropermeability to micropermeability, respectively. We have 


$\begin{array}{lcccc}\xi & R a & a & R a & a \\ 10^{-3} & 108.867 & 3.172 & 122.162 & 3.175 \\ 1.515 \times 10^{-2} & 109.254 & 3.172 & 122.778 & 3.176 \\ 2.347 \times 10^{-2} & 109.480 & 3.173 & 123.141 & 3.176 \\ 2.987 \times 10^{-2} & 109.654 & 3.173 & 123.415 & 3.176 \\ 1.316 \times 10^{-1} & 112.376 & 3.176 & 127.846 & 3.180 \\ 1 & 132.615 & 3.196 & 165.367 & 3.203\end{array}$

TABLE 7. Critical values of $R a$ and $a$ for quoted values of $\xi$, two fixed surfaces. Columns 2 and 3 are for $\lambda=0.04578, K_{r}=25$, while columns 4 and 5 are for $\lambda=0.04578$, $K r=2322$.

$\begin{array}{lcccc}\xi & R a & a & R a & a \\ 10^{-3} & 44.867 & 3.231 & 46.979 & 3.235 \\ 1.515 \times 10^{-2} & 45.352 & 3.230 & 47.564 & 3.235 \\ 2.347 \times 10^{-2} & 45.636 & 3.230 & 48.172 & 3.234 \\ 2.987 \times 10^{-2} & 45.855 & 3.230 & 48.172 & 3.234 \\ 1.316 \times 10^{-1} & 49.262 & 3.228 & 52.368 & 3.233 \\ 1 & 74.402 & 3.216 & 87.893 & 3.222\end{array}$

TABLE 8. Critical values of $R a$ and $a$ for quoted values of $\xi$, two fixed surfaces. Columns 2 and 3 are for $\lambda=0.00275, K_{r}=25$, while columns 4 and 5 are for $\lambda=0.00275$, $K r=2322$.

$\begin{array}{lcccc}\xi & a & a & a & a \\ 0 & 2.6484 & 2.6237 & 3.0684 & 3.0654 \\ 0.01 & 2.6505 & 2.6258 & 3.0690 & 3.0661 \\ 0.1 & 2.6687 & 2.6439 & 3.0746 & 3.0716 \\ 0.5 & - & - & 3.0914 & 3.0890 \\ 1 & 2.7925 & 2.7700 & 3.1034 & 3.1014\end{array}$

TABLE 9. Critical values of the wavenumber $a$ for quoted values of $\xi$, two free surfaces. Column 2 is for $\lambda=0.04578, K_{r}=25$, column 3 is for $\lambda=0.04578, K r=2322$, column 4 is for $\lambda=0.00275, K_{r}=25$ and column 5 is for $\lambda=0.00275, K r=2322$.

calculated a possible selection of values for each of these parameters which may represent real materials.

In all cases the critical Rayleigh number, $R a$, of global nonlinear stability increases as $\xi, \lambda$ or $K_{r}$ increase. Since increasing $R a$ means the layer becomes more stable, then if one is interested in insulation a higher value of $R a$ is desirable. Alternatively, if heat transfer is required we want convection to occur to aid this transfer and a smaller value of $R a$ is preferable. Thus, for insulation one should ensure $\xi, \lambda$ and $K_{r}$ are as large as possible, whereas if one requires rapid heat transfer the smallest values of these parameters are preferred.

The wavenumber behaviour is very different when fixed surface boundary conditions are employed as to when free ones are utilized. Since we expect fixed conditions in normal circumstances we deal with this. The behaviour with respect to the Brinkman coefficient is such that the critical wavenumber $a$ increases to a maximum in $\lambda$ and 
then decreases. This means that the aspect ratio of the cells decrease then increase with increasing $\lambda$.

For the values we have investigated, as $K_{r}$ increases $a$ increases, but the variation is small over the range of estimated parameter values.

The variation of $a$ in $\xi$ appears to depend critically on what values the Brinkman parameter $\lambda$ and the permeability ratio $K_{r}$ have. The non-dimensional momentum transfer coefficient $\xi$ displays both increasing or decreasing behaviour and the critical wavenumber appears to depend precisely on what values are assigned to $\lambda$ and $K_{r}$.

\section{Acknowledgements}

The work of M.G. was performed under the auspices of the National Group of Mathematical Physics GNFM-INdAM, while that of B.S. was supported by an Emeritus Fellowship of the Leverhulme Trust, EM-2019-022/9. We are indebted to four anonymous referees for their incisive critiscism of a previous version of this work. Their comments have led to very substantial improvements in the paper.

\section{Declaration of interests}

The authors report no conflict of interest.

\section{REFERENCES}

Alnofimi, K. R. \& KovsceK, A. R. 2019 Influence of microcracks on flow and storage capacities of gas shales at core scale. Trans. Porous Med. 127, 53-84.

BAnu, N. \& ReES, D. A. S. 2002 Onset of Darcy-Bénard convection using a thermal non-equilibrium model. Intl J. Heat Mass Transfer 45, 2221-2228.

BorJa, R. L., LiU, X. \& White, J. A. 2012 Multiphysics hillslope processes triggering landslides. Acta Geotechnica 7, 261-269.

Capone, F., De LuCA, R. \& Gentile, M. 2020 Coriolis effect on thermal convection in a rotating bidispersive porous layer. Proc. R. Soc. Lond. A 476, 20190875.

Chabanon, M., David, B.\& Goyeau, B. 2015 Averaged model for momentum and dispersion in hierarchical porous media. Phys. Rev. E 92, 023201.

ChandrasekHaR, S. 1981 Hydrodynamic and Hydromagnetic Stability. Dover.

CHEN, F. 1990 Throughflow effects on convective instability in superposed fluid and porous layers. J. Fluid Mech. 231, 113-133.

Chen, Z. Q., Cheng, P. \& Hsu, C. T. $2000 a$ A theoretical and experimental study on stagmant thermal conductivity of bi-dispersed porous media. Intl Commun. Heat Mass Transfer 27, 601-610.

Chen, Z. Q., Cheng, P. \& Zhao, T. S. $2000 \mathrm{~b}$ An experimental study of two phase flow and boiling heat transfer in bi-dispersed porous channels. Intl Commun. Heat Mass Transfer 27, 293-302.

Dongarra, J. J., Straughan, B. \& Walker, D. W. 1996 Chebyshev tau-QZ algorithm methods for calculating spectra of hydrodynamic stability problems. Appl. Numer. Maths 22, 399-435.

DURlofsKY, L. \& BRADY, J. F. 1987 Analysis of the Brinkman equation as a model for flow in porous media. Phys. Fluids 30, 3329-3341.

Enterria, M., Suarez-Garcia, F., Martinez-Alonso, A. \& Tascon, J. M. D. 2014 Preparation of hierarchical micro-mesoporous aluminosilicate composites by simple $\mathrm{Y}$ zeolite/MCM-48 silica assembly. J. Alloys Compounds 7, 60-69.

Falsaperla, P., Mulone, G. \& Straughan, B. 2016 Bidispersive inclined convection. Proc. R. Soc. Lond. A 472, 20160480.

Franchi, F., NibBi, R. \& Straughan, B. 2017 Modelling bidispersive local thermal non-equilibrium flow. Fluids 2, 48. 
Gelet, R., Loret, B. \& Khalili, N. 2012 Borehole stability analysis in a thermoporoelastic dual-porosity medium. Intl J. Rock Mech. Mining Sci. 50, 65-76.

Gentile, M. \& Straughan, B. $2017 a$ Bidispersive thermal convection. Intl J. Heat Mass Transfer 114, 837-840.

Gentile, M. \& Straughan, B. $2017 b$ Bidispersive vertical convection. Proc. R. Soc. Lond. A 473, 20170481.

Gerke, H. H. \& VAn Genuchten, M. T. 1993a A dual porosity model for simulating the preferential movement of water and solutes in structured porous media. Water Resour. Res. 29, 305-319.

Gerke, H. H. \& VAN Genuchten, M. T. $1993 b$ Evaluation of a first-order water transfer term for variably saturated dual-porosity flow models. Water Resour. Res. 29, 1225-1238.

Gerke, H. H. \& VAN Genuchten, M. T. 1996 Macroscopic representation of structural geometry for simulating water and solute movement in dual-porosity media. Adv. Water Resour. 19, 343-357.

Givler, R. C.\& Altobelli, S. A. 1994 A determination of the effective viscosity for the BrinkmanForcheimer flow model. J. Fluid Mech. 258, 335-370.

Guyon, E., Oger, L. \& PlonA, T. J. 1994 Transport properties in sintered porous media composed of two particle sizes. J. Phys. D: Appl. Phys. 258, 1637-1644.

Hashimoto, N. \& Smith, J. M. 1974 Diffusion in bidisperse catalyst pellets. Ind. Engng Chem. Fundam. 13, 115-120.

Homand-Etienne, F. \& Houpert, R. 1989 Thermally induced microcracking in granites: characterization and analysis. Intl J. Rock Mech. Mining Sci. 26, 125-134.

Hooman, K. \& MaAs, U. 2014 Theoretical analysis of coal stockpile self-heating. Fire Safety J. 67, 107-112.

Hooman, K., Sauret, E. \& Dahari, M. 2015 Theoretical modelling of momentum transfer function of bi-disperse porous media. Appl. Therm. Engng 75, 867-870.

ImANI, G. \& HoOman, K. 2017 Lattice Boltzmann pore scale simulation of natural convection in a differentially heated enclosure filled with a detached or attached bidisperse porous medium. Trans. Porous Med. 116, 91-113.

JiAng, T., Wang, H., Bian, X., Li, H., LiU, J., Wu, C. \& Zhou, L. 2018 Volume fracturing technology for horizontal well and its application. Lithol. Reserv. 30, 1-11.

Joseph, D. D. 1976 Stability of Fluid Motions, vol. 2. Springer.

KaWAzoe, K. \& TAKeUChI, Y. 1975 Mass transfer adsorption in bidisperse porous materials. J. Chem. Engng Japan 7, 431-437.

KIM, J. \& MORIDIS, G. J. 2015 Numerical analysis of fracture propagation during hydraulic fracturing operations in shale gas systems. Intl J. Rock Mech. Mining Sci. 76, 127-137.

KiM, S. \& Hosseini, S. A. 2015 Hydro-thermo-mechanical analysis during injection of cold fluid into a geologic formation. Intl J. Rock Mech. Mining Sci. 77, 220-236.

KUMARI, M. \& PoP, I. 2009 Mixed convection boundary layer flow past a horizontal circular cylinder embedded in a bidisperse porous medium. Trans. Porous Med. 77, 287-303.

Lin, F. C., LiU, B. H., HuAnG, C. T. \& Chen, Y. M. 2011 Evaporative heat transfer model of a loop heat pipe with bidisperse wick structure. Intl J. Heat Mass Transfer 54, 4621-4629.

MAGYARI, E. 2013 Normal mode analysis of the high speed channel flow in a bidisperse porous medium. Trans. Porous Med. 97, 345-352.

Moler, C. B.\& Stewart, G. W. 1971 An algorithm for the generalized matrix eigenvalue problem $A x=\lambda B x$. Tech. Rep. University of Texas at Austin.

Montrasio, L., Valentino, R. \& Losi, G. L. 2011 Rainfall infiltration in a shallow soil: a numerical simulation of the double-porosity effect. Electronic J. Geotech. Engng 16, 1387-1403.

MotTet, L. \& PRAT, M. 2016 Numerical simulation of heat and mass transfer in bidispersed capillary structures: application to the evaporator of a loop heat pipe. Appl. Therm. Engng 102, 770-784.

Moutsopoulos, K. N. \& Koch, D. L. 1999 Hydrodynamic and boundary-layer dispersion in bidisperse porous media. J. Fluid Mech. 385, 359-379. 
NARAsimhan, A. \& RedDY, B. V. K. $2011 a$ Laminar forced convection in a heat generating bi-disperse porous medium channel. Intl J. Heat Mass Transfer 54, 636-644.

NARASIMHAN, A. \& RedDY, B. V. K. $2011 b$ Resonance of natural convection inside a bidisperse porous medium enclosure. Trans. ASME J. Heat Transfer 133, 042601.

Narasimhan, A., Reddy, B. V. K. \& DutTA, P. 2012 Thermal management using the bi-disperse porous medium approach. Intl J. Heat Mass Transfer 55, 538-546.

NIELD, D. A. 1998 Effects of local thermal non-equilibrium in steady convection processes in saturated porous media: forced convection in a chanel. J. Porous Media 1, 181-186.

NIELD, D. A. 2000 Modelling fluid flow and heat transfer in a saturated porous medium. J. Appl. Math. Decis. Sci. 4, 165-173.

NiELD, D. A. 2016 A note on the modelling of a bidispersive porous media. Trans. Porous Med. 111, 517-520.

Nield, D. A. \& Bejan, A. 2013 Convection in Porous Media, 4th edn. Springer.

Nield, D. A. \& KuZnetsov, A. V. 2004 Forced convection in a bidisperse porous medium channel: a conjugate problem. Intl J. Heat Mass Transfer 47, 5375-5380.

Nield, D. A. \& Kuznetsov, A. V. 2005 A two-velocity temperature model for a bi-dispersed porous medium: forced convection in a channel. Trans. Porous Med. 59, 325-339.

Nield, D. A. \& Kuznetsov, A. V. $2006 a$ The onset of convection in a bidisperse porous medium. Intl J. Heat Mass Transfer 49, 3068-3074.

Nield, D. A. \& Kuznetsov, A. V. $2006 b$ Thermally developing forced convection in a bidisperse porous medium. Trans. Porous Med. 9, 325-339.

NiELD, D. A.\& KuzNetsov, A. V. 2007 The effect of combined vertical and horizontal heterogeneity on the onset of convection in a bidisperse porous medium. Intl J. Heat Mass Transfer 50, 3329-3339.

Nield, D. A. \& Kuznetsov, A. V. 2008 Natural convection about a vertical plate embedded in a bidisperse porous medium. Intl J. Heat Mass Transfer 51, 1658-1664.

Nield, D. A. \& Kuznetsov, A. V. 2011 Forced convection in a channel partly occupied by a bidisperse porous medium: symmetric case. Trans. ASME J. Heat Transfer 133, 072601.

Nield, D. A. \& Kuznetsov, A. V. 2013 A note on modelling high speed flow in a bidisperse porous medium. Trans. Porous Med. 96, 495-499.

OrszaG, S. A. 1971 Accurate solution of the Orr-Sommerfeld stability equation. J. Fluid Mech. 50, 689-703.

Patrulescu, F. O., Grosan, T. \& Pop, I. 2020 Natural convection from a vertical plate embedded in a non-Darcy bidisperse porous medium. Trans. ASME J. Heat Transfer 142, 0125504.

REES, D. A. S. 2002 The onset of Darcy-Brinkman convection in a porous layer: an asymptotic analysis. Intl J. Heat Mass Transfer 45, 2213-2220.

REES, D. A. S. 2009 Microscopic modelling of the two-temperature model for conduction in heterogeneous media: three-dimensional media. In Proceedings of the 4th International Conference on Applications of Porous Media, Istanbul, vol. 13, pp. 125-143. ICAPM.

REES, D. A. S. 2010 Microscopic modelling of the two-temperature model for conduction in heterogeneous media. J. Porous Media 13, 125-143.

Rees, D. A. S. \& BAssom, A. P. 2010 Radial injection of a hot fluid into a cold porous medium. the effects of local thermal non-equilibrium. Comput. Therm. Sci. 2, 221-230.

Rees, D. A. S., Bassom, A. P. \& Siddheshwar, P. G. 2008 a Local thermal non-equilibrium effects arising from the injection of a hot fluid into a porous medium. J. Fluid Mech. 594, 379-398.

Rees, D. A. S., Nield, D. N. \& Kuznetsov, A. V. $2008 b$ Vertical free convective boundary-layer flow in a bidisperse porous medium. Trans ASME J. Heat Transfer 130, 092601.

Revnic, C., Grosan, T., Pop, I. \& Ingham, D. B. 2009 Free convection in a square cavity filled with a bidisperse porous medium. Intl J. Therm. Sci. 48, 1876-1883.

RUbinstein, J. $1986 a$ Effective equations for flow in random porous media with a large number of scales. J. Fluid Mech. 170, 379-383. 
RUbinstein, J. $1986 b$ On the macroscopic description of slow viscous flow past a random array of spheres. J. Stat. Phys. 44, 849-863.

Scotto Di Santolo, A. \& Evangelista, A. 2008 Calibration of a rheological model for debris flow hazard mitigation in the Campania region. In Landslides and Engineered Slopes. From the Past to the Future (ed. Z. Chen, J. M. Zhang, K. Ho, F. Q. Wu \& Z. K. Li), pp. 913-919. Taylor \& Francis.

Straughan, B. 2009 On the Nield-Kuznetsov theory for convection in bidispersive porous media. Trans. Porous Med. 77, 159-168.

Straughan, B. 2015 Convection With Local Thermal Non-Equilibrium and Microfluidic Effects, Advances in Mechanics and Mathematics Series, vol. 32. Springer.

Straughan, B. 2016 Importance of Darcy or Brinkman laws upon resonance in thermal convection. Ricerche Matem. 65, 349-362.

Straughan, B. 2017 Mathematical aspects of multi-porosity continua. In Advances in Mechanics and Mathematics Series, vol. 38. Springer.

Straughan, B. 2018 Horizontally isotropic bidispersive thermal convection. Proc. R. Soc. Lond. A 474, 20180018.

Straughan, B. $2019 a$ Anisotropic bidispersive convection. Proc. R. Soc. Lond. A 475, 20190206.

Straughan, B. $2019 b$ Horizontally isotropic double porosity convection. Proc. R. Soc. Lond. A 475, 20180672.

TAQVi, S. M., Vishnoi, A. \& LeVAn, M. D. 1997 Effect of macropore convection on mass transfer in a bidisperse adsorbent particle. Adsorption 3, 127-136.

VERNESCU, B. 1990 Asymptotic analysis for an incompressible flow in fractured porous media. Intl J. Engng Sci. 28, 959-964.

WANG, K. \& LI, P. 2018 Forced convection in bidisperse porous media incorporating viscous dissipation. Appl. Therm. Engng 140, 86-94.

Wang, K., VAfai, K., LI, P. \& CEN, H. 2017 Forced convection in a bidisperse porous medium embedded in a circular pipe. Trans. ASME J. Heat Transfer 139, 102601-102694.

XIOng, X. \& ChEn, Z. M. 2019 A conjecture on the least stable mode for the energy stability of plane parralel flows. J. Fluid Mech. 881, 794-814.

Zhang, J., Li, X., Zou, X., LI, J., XIE, Z. \& Wang, F. 2019 Characterization of multi-type pore structure and fractal characteristics of the Dalong formation marine shale in Northen Sichuan basin. Energy Sources A 2009, 1-14. 